\title{
Topological structures in rough set theory: A survey
}

\author{
Pankaj Kumar Singh (D), Surabhi Tiwari*(D) \\ Department of Mathematics, Motilal Nehru National Institute of Technology Allahabad, \\ Prayagraj-211004, India
}

\begin{abstract}
Rough set theory provides a mathematical tool to study vague, imprecise, inconsistent and uncertain knowledge. The topological notions are closely related to the notions and results of rough set theory, so the conjoint study of rough set theory and topology becomes essential. Researchers have widely discussed the topological aspects and their applications in rough set theory. This study highlights the inter dependencies of topology and classical rough set theory and the significant work done in this area during the last twenty years.
\end{abstract}

Mathematics Subject Classification (2010). 54A05, 54A10, 54A40, 54D30, 54D80, 54E05, 54E17, 54F65, 54H05, 54H12, 54H20

Keywords. rough sets, topological spaces, dynamical systems

\section{Introduction}

In information science, knowledge is sometimes organized in an imprecise or vague manner and has a level of granularity. To address these issues of the knowledge system, discriminant analysis, theory of evidence (Dempster-Shafer theory), fuzzy set theory, etc. have been developed in the twentieth century. The rough set theory, introduced by Pawlak in the early 1980's [39,41], is one of them and is used as a mathematical tool for a perusal of unsettled, uncertain and vagueness of imprecise data in information systems. Rough set theory has inbuilt topological concepts. Approximation operators are the central idea in the rough set theory, and have properties of closure, interior, and frontier operators, which are topological operators induced by an equivalence relation on the universe. Thus, the study of rough set by using concepts of topology is helpful to study real-life problems like data mining [79], machine learning, pattern recognition, image processing [45], medical events [59], etc. (see also [29, 40, 47, 48, 79, 82, 83, 87]). In Pawlak's rough set theory, the equivalence relations have limitations in generating granule base (neighborhood base). Therefore there are several generalizations of Pawlak's rough set theory, and each generalization employs topological concepts. Conjoint studies of the rough set theory and topology are still needed. The methods of construction of topological structures on a universe are used in the modeling of mathematical, quantitative and qualitative data. Skowron and Wiweger first described the role of topological aspects in information system

\footnotetext{
*Corresponding Author.

Email addresses: pankaj.singh39@ymail.com; pankajsinghsahitya@gmail.com (P.K. Singh), surabhi@mnnit.ac.in; au.surabhi@gmail.com (S. Tiwari)

Received: 05.06.2018; Accepted: 04.09.2019
} 
and rough sets in $1988[56,67]$. Some of the works in the combination of rough set theory and topological theory can be found $[7,30,50,55,71,73,85]$.

The present paper outlines the significant work done by different researchers on topological structures on the rough set theory and its applications with respect to the underlying structures. Fuzzy rough sets have also been the center of attraction of many researchers but in this review article, we focus only on classical rough sets and their topological aspects.

We start the discussion from Pawlak's idea of approximation operators and then discuss more general approaches to the rough set theory and their topological aspects. Thus the sequence of the review about topological aspects of rough sets is as follows: Section 2 describes some definitions and basic concepts of topology and the rough set theory. Section 3 compiles the literature on the topological theory developed in the context of rough sets. Section 3 comprises of five subsections: In Subsection 3.1, we review the concepts of rough sets influenced by equivalence relation and their topological properties. Subsection 3.2 accumulates arbitrary binary relation based rough sets, their topological properties and results on it. Subsection 3.3 summarizes the work done in the framework of coveringbased rough sets and their related topological aspects. Subsection 3.5 recapitulate some topological aspects of logics and algebras on rough sets. Subsection 3.6 synopsizes some theories related to rough sets in three further subsections which encapsulate the discussion of the relative study of near set theory, dynamical system and formal concept analysis on the rough sets. Finally, in the last section (Section 4), we conclude the survey.

\section{Basic concepts of topological spaces and rough sets}

Throughout this paper, $U$ denotes a non-empty set, $R$ denotes an equivalence relation on $U ; \mathcal{P}(U)$ is the power set of $U$, and $X^{c}$ denotes the complement of a set $X$. In this section, we collect some basic concepts, characteristics, and operations of the rough set theory and topology.

\subsection{Topological spaces}

Definition 2.1 ([8]). A knowledge structure is a pair $(U, \tau)$, where $U$ is a non-empty set and $\tau$ is a family of subsets of $U$, containing at least $U$ and $\emptyset$. When the family $\tau$ of a knowledge structure $(U, \tau)$ is closed under union, i.e., $\cup \mathcal{F} \in \tau$ whenever $\mathcal{F} \subseteq \tau$, we say $(U, \tau)$ is a knowledge space.

Definition 2.2. Let $U$ be a non-empty set and $\tau$ a subset of the power set of $U$. Then $\tau$ is said to be a topology on $U$ if the following properties hold:

(i) $\emptyset$ and $U$ are in $\tau$.

(ii) If $A_{i} \in \tau$, where $i \in \Lambda$ (an arbitrary index set), then $\bigcup_{i \in \Lambda} A_{i} \in \tau$.

(ii) If $A_{1}, A_{2}, \ldots, A_{n} \in \tau$, then $\bigcap_{j=1}^{n} A_{j} \in \tau$.

The pair $(U, \tau)$ is said to be a topological space.

Definition 2.3. Let $(U, \tau)$ be a topological space. The members of $\tau$ are called open sets. Let $A$ be a subset of $U$. The interior of $A$ is defined as,

$$
\operatorname{int}_{\tau}(A)=\bigcup\{G \in \tau: G \subseteq A\} .
$$

Definition 2.4. Let $(U, \tau)$ be a topological space. The complement of a member of $\tau$ is called a closed set. Let $A$ be a subset of $U$. The closure of $A$ is defined as,

$$
c l_{\tau}(A)=\bigcap\{F \subseteq U: A \subseteq F \text { and } F \text { is a closed set }\} .
$$

If every member of $\tau$ is closed in a topological space $(U, \tau)$, then $\tau$ is called a quasidiscrete topology or clopen topology.

Definition $2.5([1])$. A topological space $(U, \tau)$ whose topology $\tau$ is closed under arbitrary intersections is called an Alexandrov space. 
Definition 2.6 ([76]). Let $x \in U$. A neighborhood of $x$, denoted by $N(x)$, is a subset of $U$, which contains the point $x$. A neighborhood system of $x$ is a non-empty family of neighborhoods of $x$.

Definition 2.7. Let $U$ be a non-empty set and $\mathcal{C}$ be a family of non-empty subsets of $U$. Then $\mathcal{C}$ is called a cover of $U$ if and only if $\cup \mathcal{C}=U$.

Definition 2.8. Let $\mathcal{C}$ be a covering of a non-empty set $U$. The neighborhood of any element $x \in U$ with respect to $\mathcal{C}$ is defined as,

$$
N_{\mathcal{C}}(x)=\bigcap\{A \in \mathcal{C}: x \in A\} .
$$

Definition 2.9 ([76]). Let $\Theta$ be a binary relation on a non-empty set $U$. Then we can define the predecessor neighborhood ( $\Theta$-left neighborhood) and successor neighborhood ( $\Theta$-right neighborhood) of any element $x \in U$, respectively as,

$$
\Theta_{p}(x)=\{z \in U: z \Theta x\} \text { and } \Theta_{s}(x)=\{z \in U: x \Theta z\} .
$$

\subsection{Rough set theory}

There are many approaches to the problem of how to understand and manipulate knowledge. The data table can be obtained as a result of measurements, observations or representations of the knowledge of an agent or a group of agents. Such data table is known as information systems (sometimes, knowledge representation systems or attribute-value systems) (see $[15,38])$. We can define information system as follows:

Table 1. An Information System

\begin{tabular}{|c|c|c|c|c|}
\hline$U$ & $a_{1}$ & $a_{2}$ & $\ldots$ & $a_{n}$ \\
\hline$x_{1}$ & $f\left(x_{1}, a_{1}\right)$ & $f\left(x_{1}, a_{2}\right)$ & $\ldots$ & $f\left(x_{1}, a_{n}\right)$ \\
$x_{2}$ & $f\left(x_{2}, a_{1}\right)$ & $f\left(x_{2}, a_{2}\right)$ & $\ldots$ & $f\left(x_{2}, a_{n}\right)$ \\
$x_{3}$ & $f\left(x_{3}, a_{1}\right)$ & $f\left(x_{3}, a_{2}\right)$ & $\ldots$ & $f\left(x_{3}, a_{n}\right)$ \\
$\vdots$ & $\vdots$ & $\vdots$ & $\vdots$ & $\vdots$ \\
$x_{k}$ & $f\left(x_{k}, a_{1}\right)$ & $f\left(x_{k}, a_{2}\right)$ & $\ldots$ & $f\left(x_{k}, a_{n}\right)$ \\
\hline
\end{tabular}

Definition 2.10 ([38]). An information system is a quadruple $S=(U, \mathcal{A}, V, f)$, where $U$ is a non-empty finite set, $\mathcal{A}$ is a non-empty finite set of attributes, $V=\bigcup_{a \in \mathcal{A}} V_{a}, V_{a}$ is the value set of the attribute $a, f: U \times \mathcal{A} \rightarrow V$ is defined by $f(u, a) \in V_{a}$ for all $(u, a) \in U \times \mathcal{A}$, and is called the information (knowledge) function.

Many applications require one (or more) distinguished attribute, called a decision attribute. An information system of this kind is known as a decision system and is of the form $S=(U, \mathcal{A}=C \cup D, V, f)$, where $C$ is the set of condition attributes, $D$ is the set of decision attributes, $C \cap D=\emptyset$.

The beginning pole of the rough set theory is the indiscernibility relation, which is generated by the information about objects of interest and refers to the granularity of knowledge, that affects the definition of universe of discourse. The indiscernibility relation is intended to express the fact that due to the lack of knowledge one is unable to discern some objects employing the given information.

Let $S=(U, \mathcal{A}, V, f)$ be an information system and let $\mathcal{B} \subseteq \mathcal{A}$. Then any two elements $x, y \in U$ are said to be $\mathcal{B}$-indiscernible in $S$ if and only if $f(x, a)=f(y, a)$, for each $a \in \mathcal{B}$. Clearly every subset of $\mathcal{A}$ induces a unique indiscernibilty relation which is an equivalence relation denoted by $I N D(\mathcal{B})$. In this paper, we will denote it by $R$, for convenience. The ordered pair $(U, R)$ is called an approximation space, where $R$ is an indiscernibility (equivalence) relation. 
The key point of the rough set theory is to approximate vague concepts by their lower and upper approximations, which are fundamental notions in the rough set theory. These operators form an interior and a closure of a set in the framework of partition topology. In this section, we discuss the concept of rough sets and some of their generalized structures.

Pawlak [39] defined rough sets using the concept of equivalence relation and named that relation as indiscernibility relation. Pawlak's approximation space is represented by $(U, R)$. By $[x]_{R}$, we denote the equivalence class of $R$ determined by an element $x$ of $U$.

Yao [76] generalized rough sets using arbitrary binary relations. Let $U$ be a non-empty set known as a universe, and $\Theta$ be a binary relation on $U$. Then the pair $(U, \Theta)$ is called an approximation space (Yao's approximation space).

Zhu [85] defined rough sets using coverings. Let $\mathcal{C}$ be a covering of a set $U$. The pair $(U, \mathcal{C})$ is called an approximation space (covering approximation space).

Lashin et al. [27] defined rough sets using a topological space $(U, \tau)$. In this case, if $i n t_{\tau}$ and $c l_{\tau}$ are the interior and closure operators induced by topology $\tau$, then $\left(U, i n t_{\tau}, c l_{\tau}\right)$ is an approximation space. However, in literature, $(U, \tau)$ has also been called an approximation space.

The approximation operators, lower approximation $*: \mathcal{P}(U) \rightarrow \mathcal{P}(U)$ and upper approximation ${ }^{*}: \mathcal{P}(U) \rightarrow \mathcal{P}(U)$, where $\mathcal{P}(U)$ is the power set of $U$, defined in rough set theory using various concepts are shown in the following table:

Table 2. Approximation Operators

\begin{tabular}{|c|c|c|}
\hline Case & Lower Approximation $X_{*}$ & Upper Approximation $X^{*}$ \\
\hline Equivalence Relation & $\left\{x \in U:[x]_{R} \subseteq X\right\}$ & $\left\{x \in U:[x]_{R} \cap X \neq \emptyset\right\}$ \\
Binary Relation & $\left\{x \in U: \Theta_{s}(x) \subseteq X\right\}$ & $\left\{x \in U: \Theta_{s}(x) \cap X \neq \emptyset\right\}$ \\
Covering & $\bigcup\{M \in \mathcal{C}: M \subseteq X\}$ & $X_{*} \cup\left(\bigcup\left\{N_{\mathcal{C}}(x): x \in X-X_{*}\right\}\right)$ \\
Topological Space & $\operatorname{int}_{\tau}(X)$ & $c l_{\tau}(X)$ \\
\hline
\end{tabular}

Let $\mathrm{B}(X)=X^{*}-X_{*}$ be the boundary of the set $\mathrm{X}$. If $\mathrm{B}(X)=\emptyset$, then $X$ is called a crisp or an exact set. Otherwise, $X$ is called an inexact set or a rough set.

\section{Properties of approximation operators:}

(i) $X_{*} \subseteq X \subseteq X^{*}$,

(ii) $\emptyset_{*}=\emptyset=\emptyset^{*} ; U_{*}=U=U^{*}$,

(iii) $(X \cup Y)^{*}=X^{*} \cup Y^{*}$,

(iv) $(X \cap Y)_{*}=X_{*} \cap Y_{*}$,

(v) $(X \cup Y)_{*} \supseteq X_{*} \cup Y_{*}$,

(vi) $(X \cap Y)^{*} \subseteq X^{*} \cap Y^{*}$,

(vii) $X \subseteq Y \Rightarrow X_{*} \subseteq Y_{*}$ and $X^{*} \subseteq Y^{*}$,

(viii) $\left(X^{c}\right)_{*}=\left(X^{*}\right)^{c}$ and $\left(X^{c}\right)^{*}=\left(X_{*}\right)^{c}$,

(ix) $\left(X_{*}\right)_{*}=X_{*}$ and $\left(X^{*}\right)^{*}=X^{*}$,

(x) $\left(X^{*}\right)_{*}=X^{*}$ and $\left(X_{*}\right)^{*}=X_{*}$,

(xi) $X_{*} \subseteq\left(X_{*}\right)_{*},\left(X^{*}\right)^{*} \subseteq X^{*}, X^{*} \subseteq\left(X^{*}\right)_{*},\left(X_{*}\right)^{*} \subseteq X_{*}$ and $X \subseteq\left(X^{*}\right)_{*}$.

Properties (i)-(xi) hold for Pawlak's rough sets. The properties (ii)-(viii) hold for Yao's rough sets; rest of properties do not hold in this concept, in general. All the above properties hold in Zhu's covering oriented rough sets except properties (iv) and (viii). Also, all the above properties hold in Lashin's topological approach for rough sets except properties (x) and (xi). 


\section{Topological structures on rough sets}

In this section, we discuss some variants of rough sets (and their respective topologies) given by researchers since 1996, such as, rough sets induced by equivalence relations, coverings, subsystems, arbitrary binary relations and topological spaces. We further discuss logics and algebras of rough sets. At last, we study the connection of rough set theory to the near set theory, discrete dynamical systems and formal concept analysis.

\subsection{Topological structures on rough sets generated by equivalence rela- tions}

Let $D_{R}$ be a partition of non-empty set $U$ defined by an equivalence relation $R$. It can easily be seen that the collection of all sets that can be written as unions of some members of $D_{R}$ together with the empty set is a topology on $U$. This topology is called the partition topology generated by $D_{R}$. The partition topologies are very special. They are characterized by the fact that every open set is also closed, and vice versa. Natural extensions of the partition topologies are Alexandrov topologies, which stay in one-to-one correspondence with preorders (reflexive and transitive relations).

In [31], Liu studied topological properties of the rough set theory. In this paper, the author discussed a method of defining an equivalence relation on a finite set using a given equivalence relation and a map: Let $U_{1}$ and $U_{2}$ be two non-empty sets, and $f: U_{1} \rightarrow U_{2}$ be a mapping and $R_{1}$ be an equivalence relation on $U_{1}$. Then an equivalence relation $R_{2}$ could be induced on $U_{2}$ by $R_{1}$. Let $R_{3}$ be a reflexive relation such that, if $x R_{1} y$, then $f(x) R_{3} f(y)$. Let $R_{2}$ be the transitive closure of $R_{3}$, i.e., if $m R_{3} n$, then $f^{-} 1(m) R_{2} f^{-} 1(n)$. Then $R_{2}$ is an equivalence relation on $U_{2}$ and $R_{2}=R_{3}$, if $f$ is injective. The author also proved that the mapping $f$ is continuous with respect to the topologies on $U_{1}$ and $U_{2}$ generated by their respective set of equivalence classes considered as bases. The main results proved in this paper are:

(1) If $f$ is injective and $X$ is a rough set in the approximation space $\left(U_{1}, R_{1}\right)$, then $f(X)$ is a rough set in the approximation space $\left(U_{2}, R_{2}\right)$.

(2) If $f$ is surjective and $Y$ is a rough set in the approximation space $\left(U_{2}, R_{2}\right)$, then $f^{-1}(Y)$ is a rough set in the approximation space $\left(U_{1}, R_{1}\right)$.

(3) If $f$ is bijective, then $f$ is continuous and open.

By means of the topological properties of a map $f$, we can describe some vague objects of an information system.

Some applications of topological structures on rough sets in the area of image processing was studied by Quing et al. in [73]. In this paper, topology has been introduced on a subset $X$ of $U$ instead of the universe $U$.

Definition 3.1. Let $X=\left(X_{*}, X^{*}\right)$ be a rough set of the Pawlak's approximation space $(U, R)$. Let $\tau_{*}$ and $\tau^{*}$ be any two topologies which contain only exact subsets of $X_{*}$ and $X^{*}$ respectively. Then the pair $\tau=\left(\tau_{*}, \tau^{*}\right)$ is said to be a rough topology on the rough set $X=\left(X_{*}, X^{*}\right)$ and the pair $(X, \tau)$ is known as a rough topological space. Further, for a rough topology $\tau=\left(\tau_{*}, \tau^{*}\right), \tau_{*}$ is known as the lower rough topology and $\tau^{*}$ is known as the upper rough topology on $X$.

Consider a rough topological space $(X, \tau)$, where $X=\left(X_{*}, X^{*}\right)$ and $\tau=\left(\tau_{*}, \tau^{*}\right)$. Let $T$ be the collection of all rough open subsets of $(X, \tau)$. Then $T$ is a topology on $X$.

Definition 3.2. Let $X=\left(X_{*}, X^{*}\right)$ and $Y=\left(Y_{*}, Y^{*}\right)$ be two rough sets. Assume that $f_{1}: X_{*} \rightarrow Y_{*}$ and $f_{2}: X^{*} \rightarrow Y^{*}$ are bijective maps. At the same time, $f_{1}$ and $f_{2}$ keep the properties of an open set and a closed set; and closure is invariable, i.e., $f_{1}(c l(A))=$ $\operatorname{cl}\left(f_{1}(A)\right)$ and $f_{2}(\operatorname{cl}(A))=\operatorname{cl}\left(f_{2}(A)\right), A \subseteq X ; \operatorname{cl}(A)$ is closure of $A$ in $X$. Then $f_{1}$ is called a homeomorphism from $X_{*}$ to $Y_{*}$, and $f_{2}$ is called a homeomorphism from $X^{*}$ to $Y^{*}$. 
Further, $f_{1}$ and $f_{2}$ are also called the lower rough homeomorphism and the upper rough homeomorphism from $X$ to $Y$, respectively. Moreover, $f=\left(f_{1}, f_{2}\right)$ is called a rough homeomorphism from $X$ to $Y$ and is labelled as $f: X \rightarrow Y$.

Definition of compact rough topological space is also introduced and some basic results on it are proved, like homeomorphic image of a compact space is compact, etc.

Definition 3.3. Let $X=\left(X_{*}, X^{*}\right)$ be a rough set. For any open covering $\mathcal{U}_{1}$ of the set $X_{*}$, if there is always a finite subcover $\mathcal{U}_{1}^{0}$, then $X_{*}$ is called the compact set or the compact lower approximation of $X$. At the same time, for any open covering $\mathcal{U}_{2}$ of the set $X^{*}$, if there also is always a finite subcover $\mathcal{U}_{2}^{0}$, then $X^{*}$ is called the compact set or the compact upper approximation of $X$. The ordered pair $\left(\mathcal{U}_{1}, \mathcal{U}_{2}\right)$ is called an open covering of $X$. If there is always a finite subcover ordered pair $\left(\mathcal{U}_{1}^{0}, \mathcal{U}_{2}^{0}\right)$ of $\left(\mathcal{U}_{1}, \mathcal{U}_{2}\right)$, then $X$ is called a compact rough topological space.

The authors have discussed applications of this topological structure to some real life problems such as finger print recognition, reduction of attributes etc. Let us discuss the application to reduction of attributes: The topological properties (such as finite coverage characteristic) provides the ability to analyse an unnecessary attribute of information systems. For example, assume that $S=(U, \mathcal{A}, V, f)$ is an information system. The equivalence relation, labeled $R_{\mathcal{B}}$, is induced by the attribute set $\mathcal{B} \subseteq \mathcal{A}$. Let $\mathcal{U}_{R}=\left\{V_{a_{i}}\right\}$ be a coverage of the relation $R_{\mathcal{A}}$, for all $V_{a_{i}} \in \mathcal{U}_{R}, i \in\{1,2, \ldots\}$. If $\left(\mathcal{U}_{R} \backslash\left\{V_{a_{i}}\right\}\right)_{R}$ is still a coverage of the relation $R_{\mathcal{A}}$, then the attribute $V_{a_{i}}$ is unnecessary. If there are no unnecessary attributes in the system, the attribute set $\mathcal{A}$ is independent. The subset $\mathcal{B} \subseteq \mathcal{A}$ is called a reduction of $\mathcal{A}$, usually labeled as $\operatorname{red}(\mathcal{A})$, if $\mathcal{U}_{R}$ covers $R_{\mathcal{A}}$ and $R_{\mathcal{B}}$, and there are no unnecessary attributes in $\mathcal{B}$.

For example, assume that $S=(U, \mathcal{A}, V, f)$, where $U=\left\{x_{1}, x_{2}, \ldots, x_{8}\right\}$, the attribute set $\mathcal{A}=\left\{c_{1}, c_{2}, c_{3}, c_{4}\right\}, V_{1}=V_{2}=V_{3}=\{1,2,3\}, V_{4}=\{1,2\}$, and the information function $f$ is shown in Table 3. Obviously,

Table 3. Information System

\begin{tabular}{|c|c|c|c|c|}
\hline$U$ & $c_{1}$ & $c_{2}$ & $c_{3}$ & $c_{4}$ \\
\hline$x_{1}$ & 1 & 1 & 1 & 1 \\
$x_{2}$ & 1 & 2 & 2 & 1 \\
$x_{3}$ & 1 & 1 & 1 & 1 \\
$x_{4}$ & 1 & 2 & 2 & 1 \\
$x_{5}$ & 2 & 2 & 1 & 1 \\
$x_{6}$ & 2 & 2 & 1 & 1 \\
$x_{7}$ & 3 & 3 & 3 & 2 \\
$x_{8}$ & 3 & 3 & 3 & 2 \\
\hline
\end{tabular}

Table 4. Information Form of Compression

\begin{tabular}{|c|c|c|c|c|}
\hline$U$ & $c_{1}$ & $c_{2}$ & $c_{3}$ & $c_{4}$ \\
\hline$\left(x_{1} ; x_{3}\right)$ & 1 & 1 & 1 & 1 \\
$\left(x_{2} ; x_{4}\right)$ & 1 & 2 & 2 & 1 \\
$\left(x_{5} ; x_{6}\right)$ & 2 & 2 & 1 & 1 \\
$\left(x_{7} ; x_{8}\right)$ & 3 & 3 & 3 & 2 \\
\hline
\end{tabular}

Table 5. Most Concise Reduction

\begin{tabular}{|c|c|}
\hline$c_{1}$ & $c_{2}$ \\
\hline 1 & 1 \\
1 & 2 \\
2 & 2 \\
3 & 3 \\
\hline
\end{tabular}

\begin{tabular}{|c|c|}
\hline$c_{1}$ & $c_{3}$ \\
\hline 1 & 1 \\
1 & 2 \\
2 & 1 \\
3 & 3 \\
\hline
\end{tabular}

\begin{tabular}{|c|c|}
\hline$c_{2}$ & $c_{3}$ \\
\hline 1 & 1 \\
2 & 2 \\
2 & 1 \\
3 & 3 \\
\hline
\end{tabular}

$\mathcal{A}_{c_{1}}=\left\{\left\{x_{1}, x_{2}, x_{3}, x_{4}\right\},\left\{x_{5}, x_{6}\right\},\left\{x_{7}, x_{8}\right\}\right\}$ 


$$
\begin{gathered}
\mathcal{A}_{c_{2}}=\left\{\left\{x_{1}, x_{3}\right\},\left\{x_{2}, x_{4}, x_{5}, x_{6}\right\},\left\{x_{7}, x_{8}\right\}\right\}, \\
\mathcal{A}_{c_{3}}=\left\{\left\{x_{1}, x_{3}, x_{5}, x_{6}\right\},\left\{x_{2}, x_{4}\right\},\left\{x_{7}, x_{8}\right\}\right\}, \\
\mathcal{A}_{c_{4}}=\left\{\left\{x_{1}, x_{2}, x_{3}, x_{4}, x_{5}, x_{6}\right\},\left\{x_{7}, x_{8}\right\}\right\} .
\end{gathered}
$$

Based on the above formulae,

$$
U \backslash \mathcal{A}=\left\{\left\{x_{1}, x_{3}\right\},\left\{x_{2}, x_{4}\right\},\left\{x_{5}, x_{6}\right\},\left\{x_{7}, x_{8}\right\}\right\} .
$$

After compressing the objects and their informations, Table 4 is obtained. Since the combination of formulae (3.1)-(3.4) covers (3.5) and the combination of formulae (3.1)-(3.3) also covers (3.5), therefore the attribute $c_{4}$ is an unnecessary attribute in the information system represented by Table 3 (or Table 4). Similarly, by the compactness property, we can obtain the three simplest reductions of attributes from this information: $\left\{c_{1}, c_{2}\right\}$, $\left\{c_{1}, c_{3}\right\}$, and $\left\{c_{2}, c_{3}\right\}$. Accordingly, the three most concise reduction forms of information systems, shown in Table 5, can be obtained.

The study of mathematical structures of rough sets in infinite universes of discourse has been done in [72]. It is well known that it is often easier to deal with compact sets and continuous functions defined on compact sets have more controlled behavior than functions on non-compact sets. For example, two-dimensional compact surfaces have a nice classification theorem. Also, classifying non-compact surfaces is more difficult and less satisfying. If space is not compact, we can make it compact by the compactification of the space. In the field of image analysis, classification of images of a compact universe become easier. In [13] Hajri et al., studied the compactification of a scattered space. A topological space $U$ is said to be scattered if every non-empty subset $X$ of $U$ contains at least one isolated point. Digital line and digital plane are examples of scattered spaces. Hajri et al. have employed this theory of compactification of scattered spaces in the classification and regularization of digital images.

In [33], Mathew and John enhanced the results of [73]. According to the definition of open sets given in [73], $\emptyset=(\emptyset, \emptyset)$ is not always open because $\emptyset \subseteq X_{*} \subseteq \emptyset \subseteq X^{*}$ is not true for a rough set $X=\left(X_{*}, X^{*}\right)$ with non-empty lower approximation $X_{*}$. Thus $\emptyset$ is not always open and therefore, the basic definition of topology in [73] is not valid. In the process to rectify this problem, they defined a new topological structure on rough sets to create a rough topological space.

Another application of the concept of topology in rough sets is to study symptoms of diseases like Chikungunya and Diabetes [59]. The authors showed that the topology induced by approximation operations can be used used to deal with real world problems. The concept of topological basis induced by a rough set model has been applied to find the deciding factors of a recent outbreak in Chikungunya and Diabetes. The following algorithm has been used to find the deciding factors of the diseases as mentioned above:

Step I: Let $U$ be a finite universe. A finite set $\mathcal{A}$ of attributes is divided into two classes, $C$ of condition attributes and $D$ of decision attributes. An equivalence relation $R$ on $U$ is defined with respect to $C$. A subset $X$ of $U$ represent the data as an information table, columns of which are labeled by attributes, rows by objects and entries of the table are attribute values.

Step II: Find the lower approximation, upper approximation and the boundary region of $X$ with respect to $R$.

Step III: Generate the topology $\tau_{R}$ on $U$ and its basis $\beta_{R}$ as: $\tau_{R}=\left\{\emptyset, U, X^{*}, X_{*}, \mathrm{~B}(X)\right\}$ and $\beta_{R}=\left\{U, X_{*}, \mathrm{~B}(X)\right\}$, where $\mathrm{B}(X)$ is the boundary of the set $X$.

Step IV: Remove an attribute ' $a$ ' from $C$ and find the lower and upper approximations and the boundary region of $X$ with respect to the equivalence relation on $C-(a)$.

Step V: Generate the topology $\tau_{R-(a)}$ on $U$ and its basis $\beta_{R-(a)}$.

Step VI: Repeat steps III and IV for all attributes in $C$.

Step VII: Those attributes in $C$ for which $\beta_{R-(a)} \neq \beta_{R}$ form the core $(R)$. 
Step VIII: The attributes of $\operatorname{core}(R)$ are the deciding factors of the diseases Diabetes and Chikungunya.

Till 1996, the concept of rough distance function was unknown. Biswas [6] introduced the concept of rough metric space, and some basic terms of rough metric space like rough diameter, rough open balls, rough interior points, rough open sets, etc.

Definition 3.4. Let $X$ be a non-empty rough subset of an Pawlak's approximation space $(U, R)$. Then the function $d: X \times X \rightarrow \mathbb{R}$ is called a rough metric on $X$ if the following conditions are true, for all $x, y, z \in X$,

(i) $d(x, y) \geq 0$,

(ii) $d(x, y)=0$ if and only if $[x]_{R}=[y]_{R}$,

(iii) $d(x, y)=d(y, x)$,

(iv) $d(x, y)+d(y, z) \geq d(x, z)$.

The function ' $d$ ' is a rough metric or a rough distance function on $X$, and the pair $(X, d)$ is a rough metric space. The rough distance between $x$ and $y$ is defined as $d(x, y)$.

Since rough open balls are not rough sets, therefore rough open sets are not rough sets, in general.

In [70], Wolski discussed another topological property, viz. completion of a perceptual system in the near set framework and introduced the concept of a separating completion.

Definition 3.5. A perceptual system is a pair $(U, \Phi)$, where $U$ is a non-empty finite set of perceptual objects and $\Phi$ is a countable set of probe functions $\phi_{i}: U \rightarrow \mathbb{R}$.

Probe functions are real valued functions which represent features of a physical object, a simple examples of probe functions are the size or weight of an object. Any probe function can induce a pseudometric (see [43]).

Definition 3.6. A family $\mathcal{D}=\left\{d_{i}: i \in I\right\}$ of pseudometrics on $U$ is called separating if, for each pair of points $x \neq y$, there exists $d_{i} \in \mathcal{D}$ such that $d_{i}(x, y) \neq 0$.

Let $(U, \Phi)$ be a perceptual system, then the perceptual indiscernibility relation $R_{\Phi}$ is defined as $R_{\Phi}=\left\{(x, y) \in U \times U\right.$ : for all $\left.\phi_{i} \in \Phi, \phi_{i}(x)-\phi_{i}(y)=0\right\}$ and $\mathcal{D}(\Phi)=\left\{d_{\phi_{i}}\right.$ : $\left.\phi_{i} \in \Phi\right\}$, where $d_{\phi_{i}}(x, y)=\left|\phi_{i}(x)-\phi_{i}(y)\right|$ is a family of pseudometrics which usually does not separate all points of $U$.

Definition 3.7. Let $(U, \Phi)$ be a perceptual system such that $\mathcal{D}(\Phi)$ is not a separating family on $U$. Then a tolerance (reflexive and symmetric) relation $\mu$ on $U$ satisfying:

if $x R_{\Phi} y$ and $x \mu y$, then $x=y$, for all $x, y \in U$,

will be called a separating completion of $\mathcal{D}(\Phi)$.

When one deals with an equivalence relation $R$ on $U$, one is interested in the canonical projection $p: U \rightarrow U / R$, such that $p(x)=[x]_{R}$, where $U / R$ is set of all equivalence classes induced by relation $R$.

Proposition 3.8. Let $(U, \Phi)$ be a given perceptual system, such that $\mathcal{D}(\Phi)$ is not separating family on $U$, and let $\mu$ be a separating completion of $\mathcal{D}(\Phi)$. Then for each class $X$ of $\mu$, the restriction of $p: U \rightarrow U / R_{\Phi}$ to $X$, is one-one correspondence.

The author showed that how completion could be used to provide a topology on the family of perceptual elementary sets, i.e., a higher order granulation of objects.

Kondo and Dudek [26] studied the fundamental topological properties of rough sets induced by equivalence relations and proved that an Pawlak's approximation space $(U, R)$ is strongly connected, i.e., the whole space is a single granule if and only if $R=U \times U$. This result can be used to characterize the freeness of a subset $A$ of $U$ : A subset $A$ of $U$ is free if every element in $A$ is related to itself only, i.e., $A$ is free if and only if $x \notin(A-\{x\})^{*}$, for all $x \in A$. Moreover, $A$ is free if and only if $R \cap(A \times A)=\{(x, x): x \in A\}$. 


\subsection{Rough set theory obtained by arbitrary binary relations}

For an arbitrary binary relation, the successor elements (or right neighborhood, see Definition 2.9) of a given element are precisely its neighborhood.

As discussed earlier, generalization of Pawlak's rough set concept was needed due to the limitation of equivalence classes in generating neighborhood base. This was done by Yao in where he studied the approximation operators in terms of neighborhood operators: Let $U$ be a non-empty set. For each element $x$ of $U, n(x) \subseteq U$ is called a neighborhood of $x$, where $n$ is a mapping from $U$ to $\mathcal{P}(U)$. A neighborhood of $x$ need not contain $x$. The neighborhood operator $n$ is said to be a reflexive operator if for all $x \in U, x \in n(x)$; a symmetric operator if for all $x, y \in U, x \in n(y) \Rightarrow y \in n(x)$; and a transitive operator if for all $x, y, z \in U, y \in n(x)$ and $z \in n(y) \Rightarrow z \in n(x)$. A neighborhood system $N S(x)$, of an element $x$ is a family of neighborhoods of $x$. Neighborhood system of $U$, denoted by $N S(U)$, is the collection of $N S(x)$, for all $x \in U$. The author considered a special type of neighborhood system called 1-neighborhood system, in which each element has exactly one neighborhood. For a finite universe $U$, we can extend a neighborhood operator $n$ from $U$ to $\mathcal{P}(U)$ as follows:

$$
N(X)=\bigcup_{x \in X} n(x) .
$$

For the empty set $\emptyset, N(\emptyset)=\emptyset$. The mapping $N: \mathcal{P}(U) \rightarrow \mathcal{P}(U)$ associates each subset of $U$ with a subset of $U$. The class of 1-neighborhood systems can be interpreted using the more familiar notion of binary relations. For a binary relation $\Theta$ and two elements $x, y \in U$, if $x \Theta y$, we say that $y$ is $\Theta$-related to $x, x$ is a predecessor of $y$ and $y$ is a successor of $x$. Given a binary relation $\Theta$, define

$\Theta_{s}(x)=\{y: x \Theta y\}$,

$\Theta_{p}(x)=\{y: y \Theta x\}$

$\Theta_{p \wedge s}(x)=\{y: x \Theta y$ and $y \Theta x\}=\Theta_{p}(x) \cap \Theta_{s}(x)$,

$\Theta_{p \vee s}(x)=\{y: x \Theta y$ or $y \Theta x\}=\Theta_{p}(x) \cup \Theta_{s} x$.

Then $\Theta_{s}, \Theta_{p}, \Theta_{p \wedge s}$ and $\Theta_{p \vee s}$ are neighborhood operators from $U$ to $\mathcal{P}(U)$. Yao proved that these neighborhoods generate same upper and lower approximations if and only if the binary relation $\Theta$ is symmetric. The author, therefore, used an arbitrary neighborhood operator $n$ to define distinct pairs of approximation operators as follows:

(I). $X_{*}=\{x \in U: n(x) \subseteq X\}, X^{*}=\{x \in U: n(x) \cap X \neq \emptyset\}$.

(II). $X_{*}=\cup\{n(x): x \in \bar{U}, n(x) \subset X\}=\{x \in U$ : there exists $y$ such that $x \in n(y) \subseteq$ $X\}, X^{*}=\left(\left(X^{c}\right)_{*}\right)^{c}=\{x \in U$ : for all $y, x \in n(y)$ implies $n(y) \cap X \neq \emptyset\}$.

(III). $X^{*}=\cup\{n(X): x \in U, n(x) \cap X \neq \emptyset\}=\{x \in U$ : there exists $y$ such that $x \in n(y) \cap X \neq \emptyset\}, X_{*}=\left(\left(X^{c}\right)^{*}\right)^{c}=\{x \in U$ : for every $y, x \in n(y)$ implies $n(y) \subseteq X\}$.

Note that in $[\mathrm{I}]$, the approximations are element based. Lower approximation in [II] and upper approximation in [III] are granule based definitions and these are not dual of each other, in general. Thus to obtain of duality between the upper and lower approximation operators, Yao defined the upper approximation in [II] and lower approximation in [III] separately using the set theoretic complementation ' ()$^{c}$ '. Conditions on neighborhood operators under which some or all of these approximation operators are equivalent are given in the following theorem.

Theorem 3.9. Let $U$ be a non-empty set and $n: U \rightarrow \mathcal{P}(U)$ be a neighborhood operator. Then,

(1) The two pairs of lower and upper approximation operators defined by $[I I]$ and $[I I I]$ are equivalent if and only if the family $\{n(x) \neq \emptyset: x \in U\}$ forms a partition of the universe $U$. 
(2) The two pairs of lower and upper approximation operators defined by $[I]$ and $[I I]$ are equivalent if and only if the neighborhood operator $n$ is reflexive and transitive.

(3) The two pairs of lower and upper approximation operators defined by $[I]$ and $[I I I]$ are equivalent if and only if the neighborhood operator $n$ is symmetric and transitive.

(4) The three pairs of lower and upper approximation operators defined by $[I],[I I]$ and $[I I I]$ are equivalent if and only if $n$ is reflexive, symmetric and transitive.

Kondo, in [25] studied the topological properties of generalized rough sets by using an arbitrary reflexive binary relation instead of an equivalence relation. A type of compactness condition $C O M P$ was proposed, and it was proved that a topology which satisfies COMP could determine the lower and upper approximation operators induced by reflexive and transitive relations. The condition $C O M P$ is: For all $O_{\lambda} \in \tau$ and $A \subseteq U$, if $\cap O_{\lambda} \cap A=\emptyset$, then there exists a finite subset $\left\{O_{i}\right\}(i \leqslant n)$ of $\left\{O_{\lambda}\right\}$ such that $O_{1} \cap \ldots \cap O_{n} \cap A=\emptyset$, where $\tau$ is the topology on $U$.

This compactness condition is not satisfied by the topology induced by a reflexive and transitive relation. Hence Qin et al. in [51] proposed another kind of compactness condition on the topology generated by the approximation operators induced by a reflexive and transitive relation:

$C O M P^{\prime}:$ For all $x \in U$ and $A \subseteq U$, if $x \in \operatorname{cl}(A)$, then there exists $y \in A$ such that $x \in \operatorname{cl}(\{y\})$, where $(U, \tau)$ is topological space and $c l$ is a closure operator on $U$.

There is a one-to-one correspondence between the set of all reflexive and transitive relations and the set of all topologies which satisfy $\left(C O M P^{\prime}\right)$. This correspondence is of theoretical significance for the study of axiomatic characterization of rough approximation operators on an infinite universe. Further relaxing the conditions on the indiscernibility relation, Li et al. [29] investigated properties of the topological spaces induced by reflexive relations or tolerance relations on the universe such as separating property, countable property, local connectedness and pseudo-metrizability. The necessary and sufficient condition for a topological space $(U, \tau)$ to be an approximation space is that $(U, \tau)$ is both pseudo-metrizable and pseudo-discrete.

In the above discussion, we considered the topological structures induced by the elements based approximation operators defined in [I]. Zhang et al. [81] studied topological structure of relation based generalized approximation operators, defined as above in [II] and [III]. Let $\Theta$ be a binary relation on $U$. The necessary and sufficient condition for the upper approximation operator to be a topological closure and pseudo closure operator and the lower approximation operator to be an interior operator is that $\Theta$ is reflexive and transitive.

In [27], Lashin et al. studied the rough set theory for topological spaces, especially for the class of clopen topologies in which every open set is closed. Topological spaces are approximation spaces generated by a binary relation if the collection of right neighborhoods is taken as a subbase.

\subsection{Topology on covering based rough sets}

Covering based rough sets are natural extensions of classical rough sets $[4,78,80,86]$. Zhu [85] pursued generalization of rough set theory by using covers in place of equivalence classes. Recall the definition of lower and upper approximation of covering based rough sets: Let $U$ be a non-empty set and $\mathcal{C}$ be cover of $U$. The pair $(U, \mathcal{C})$ is called a covering approximation space. For any $X \subseteq U$, the lower approximation $X_{*}=\bigcup\{M \in \mathcal{C}: M \subseteq X\}$ and the upper approximation $X^{*}=X_{*} \cup\left\{N_{\mathcal{C}}(x): x \in X-X_{*}\right\}$, where $N_{\mathcal{C}}(x)=\bigcap\{M \in$ $\mathcal{C}: x \in M\}$. In covering based approximation spaces, the upper approximation satisfies closure properties but the lower approximation does not satisfies interior properties, as property (iv) of approximation operators, i.e., $(X \cap Y)_{*}=X_{*} \cap Y_{*}$ does not holds for 
covering based rough sets discussed, in general (see [85]). To resolve this problem, the author found a characterization for lower approximation to behave like interior operator: "Lower approximation operator is an interior operator if and only if for every $M, N \in \mathcal{C}$, $M \cap N=\emptyset$ or $M \cap N$ is a union of elements of $\mathcal{C}$, where $\mathcal{C}$ is a cover of the a non-empty set $U$." The author also studied the properties of reduct $[84,86]$, that is, under which conditions a cover $\mathcal{C}$ is reducible.

Definition 3.10. Let $\mathcal{C}$ be a covering of a non-empty set $U$ and $M \in \mathcal{C}$. If $M$ can be represented as a union of elements of $\mathcal{C}-\{M\}$, then we say that $M$ is reducible in $\mathcal{C}$, otherwise $M$ is irreducible. If there exists an element $K \in \mathcal{C}$, which is reducible, then we say that $\mathcal{C}$ is reducible.

Definition 3.11. Given a covering $\mathcal{C}$ of a non-empty set $U$. By eliminating all reducible elements from $\mathcal{C}$, one finds an irreducible covering, called a reduct of $\mathcal{C}$ which is denoted by $\operatorname{reduct}(\mathrm{C})$.

The covering $\mathcal{C}$ and the reduct $(\mathcal{C})$ both generate the same upper and lower approximations. The two covering $\mathcal{C}_{1}$ and $\mathcal{C}_{2}$ generate the same lower approximation if and only if their reducts are same, that is, $\operatorname{reduct}\left(\mathrm{C}_{1}\right)=\operatorname{reduct}\left(\mathrm{C}_{2}\right)$. Further, if $\operatorname{reduct}\left(\mathrm{C}_{1}\right)=$ reduct $\left(\mathcal{C}_{2}\right)$, then they generate same upper approximations. Also, $\mathcal{C}$ generates Pawlak's approximation operators if and only if $\operatorname{reduct}(\mathrm{C})$ is a partition of the universe $U$. The lower and upper approximation operators of $U$ can be axiomatized as follows:

Theorem 3.12. Let $U$ be a non-empty set. If an operation $L: \mathcal{P}(U) \rightarrow \mathcal{P}(U)$ satisfies the following properties: for any $X, Y \subseteq U$,

(i) $L(U)=U$,

(ii) $X \subseteq Y \Rightarrow L(X) \subseteq L(U)$,

(iii) $L(X) \subseteq X$,

(iv) $L(L(X))=L(X)$,

then there exists a covering $\mathcal{C}$ of $U$ such that the lower approximation operation of $X$, i.e., $X_{*}$, generated by $\mathrm{C}$, equals to $L(X)$.

Theorem 3.13. Let $U$ be a non-empty set. If an operation $H: \mathcal{P}(U) \rightarrow \mathcal{P}(U)$ is a closure operator, i.e., $H$ satisfies the following properties: for any $X, Y \subseteq U$,

(i) $H(X \cup Y)=H(X) \cup H(Y)$,

(ii) $X \subseteq H(X)$,

(iii) $H(\emptyset)=\emptyset$,

(iv) $H(H(X))=H(X)$,

then there exists a covering $\mathcal{C}$ of $U$ such that the upper approximation operation of $X$, i.e., $X^{*}$, generated by, $\mathcal{C}$ equals to $H(X)$.

In [88], Zhu and Wang discussed topological properties of four types of covering generalized rough sets. The conditions under which lower approximation operations become interior operators; and the conditions under which the upper approximation operations become closure operators are also studied.

Definition 3.14. Let $(U, \mathcal{C})$ be a covering approximation space and $x \in U$. Then $\operatorname{Md}(x)=\{K \in \mathcal{C}: x \in K$ and (for all $S \in \mathcal{C}$ and $x \in S$ and $S \subseteq K \Rightarrow K=S$ ) $\}$ is called the minimal description of $x$ with respect to the covering $\mathcal{C}$.

Definition 3.15. Let $(U, \mathcal{C})$ be a covering approximation space and $x \in U$. Then $\bigcup\{K$ : $x \in K \in \mathcal{C}\}$ is called the indiscernible neighborhood of $x$ and is denoted by Friends $(x)$. For $x \in U, \bigcup\{\operatorname{Md}(x)\}$ is called the closed friend of $x$ and denoted as CFriends $(x)$.

Definition 3.16. Let $\mathcal{C}$ be a covering of $U$. Then the operation $\mathbf{C L}$ is defined as follows, for $X \subseteq U$, 
$\mathbf{C L}(X)=\bigcup\{K: K \in \mathcal{C}, K \subseteq X\}$.

We call $\mathbf{C L}$ the covering lower approximation operation.

Definition 3.17. Let $\mathcal{C}$ be a covering of $U$. Then the operations $\mathbf{F H}, \mathbf{S H}, \mathbf{T H}$ and $\mathbf{R H}$ : $\mathcal{P}(U) \rightarrow \mathcal{P}(U)$ are defined as follows, for $X \subseteq U$,

$$
\begin{gathered}
\mathbf{F H}(X)=\mathbf{C L}(X) \cup\{\operatorname{Md}(x): x \in X-\mathbf{C L}(X)\}, \\
\mathbf{S H}(X)=\bigcup\{K: K \in \mathcal{C}, K \cap X \neq \emptyset\}, \\
\mathbf{T H}(X)=\bigcup\{\operatorname{Md}(x): x \in X\}, \\
\mathbf{R H}(X)=\mathbf{C L}(X) \cup\{K: K \in \mathcal{C}, K \cap(X-\mathbf{C L}(X)) \neq \emptyset\} .
\end{gathered}
$$

We call $\mathbf{F H}, \mathbf{S H}, \mathbf{T H}$, and $\mathbf{R H}$ the first, second, third and fourth type of covering upper approximation operations, respectively.

The conditions under which $\mathbf{C L}$ is an interior operator and $\mathbf{F H}, \mathbf{S H}, \mathbf{T H}$, and $\mathbf{R H}$ become closure operators are:

(1) $\mathbf{C L}$ is interior operator, $\mathbf{R H}$ and $\mathbf{F H}$ are closure operators if and only if for all $K_{1}, K_{2} \in \mathcal{C}, K_{1} \cap K_{2}$ is union of finite elements in $\mathcal{C}$.

(2) SH is a closure operator if and only if for all $x \in U$ and $K \in \mathcal{C}$, either $K \subseteq$ Friends $(x)$ or $K \cap$ Friends $(x)=\emptyset$.

(3) $\mathbf{T H}$ is a closure operator if and only if for all $x \in U$ and $K \in \mathcal{C}$, either $K \subseteq$ Friends $(x)$ or $K \cap$ Friends $(x)=\emptyset$.

Further RH is a closure operator if and only if $\mathbf{C L}$ is an interior operator.

Some more approximation spaces were defined by Yang et al. in [74]. The three upper approximation operators $\mathbf{I H}, \mathbf{X H}$ and $\mathbf{V H}$ corresponding to three approximation spaces are:

$$
\begin{gathered}
\mathbf{I H}(X)=\mathbf{C L}(X) \cup\left\{N_{\mathcal{C}}(x): x \in X-\mathbf{C L}(X)\right\}=\bigcup\left\{N_{\mathcal{C}}(x): x \in X\right\}, \\
\mathbf{X H}(X)=\left\{x: N_{\mathcal{C}}(x) \cap X \neq \emptyset\right\}, \\
\mathbf{V H}(X)=\bigcup\left\{N_{\mathcal{C}}(x): N_{\mathcal{C}}(x) \cap X \neq \emptyset\right\} .
\end{gathered}
$$

Let $\mathcal{C}$ be a covering of $U, \tau_{\mathcal{C}}$ be the topology induced by $\mathcal{C}$. Then $\mathcal{B}$ (arbitrary base of the topology $\tau_{\mathcal{C}}$ )and $\mathcal{C}$ generate the same set approximations based on $\mathbf{I H}, \mathbf{X H}$ and $\mathbf{V H}$ types of covering rough sets if and only if $\mathcal{B}$ is a subbase of $\tau_{\mathcal{C}}$. Let $\mathcal{C}^{\prime} \subseteq \mathcal{C}$ and $\tau_{\mathcal{C}}$ be the topology induced by $\mathcal{C}$. Then $\mathcal{C}^{\prime}$ is an granular reduct of $\mathcal{C}$ based on $\mathbf{I H}, \mathbf{X H}$ and $\mathbf{V H}$ types of covering rough sets if and only if $\mathcal{C}^{\prime}$ is a minimal subbase of $\tau_{\mathcal{C}}$.

Another contribution to covering-based rough sets was done by Thuan in [60], Thuan studied the topology constructed by family of covering and investigated its properties: Let $S=(U, \mathcal{A}, V, f)$ be an information system. Let $a \in \mathcal{A}$ and $\Theta_{a}$ be a binary relation defined as follows:

$$
x \Theta_{a} y \text { if and only if } a(x) \cap a(y) \neq \emptyset .
$$

Clearly the relation $\Theta_{a}$ is reflexive. With this definition, $\Theta_{a}$ determines a cover $C_{a}=$ $\left\{\Theta_{a}(x): x \in U\right\}$ of $U$, where $\Theta_{a}(x)=\left\{y \in U: x \Theta_{a} y\right\}$. Also, $\Theta_{a}$ determines a topology $\tau_{a}$ : With respect to $\Theta_{a}$, we can define the $\Theta_{a}$-left and $\Theta_{a}$-right neighborhoods (see Definition 2.9 ) of an element $x \in U$. To construct the topology $\tau_{a}$ using $\Theta_{a}$-right neighborhoods, we consider the family $S_{a}=\left\{\Theta_{a}(x): x \in U\right\}$ as the subbase. Similarly, we can construct the topology $\tau_{a}$ using $\Theta_{a}$-left neighborhoods. For all attributes in $A$, we get the topology $\tau_{S}$ which is induced by the subbase $\bigcup_{a \in A} S_{a}$, where $S_{a}$ is a subbase of topology $\tau_{a}$. Thus the topological space $\left(U, \tau_{S}\right)$ is constructed from a family of cover $\left\{C_{a}\right.$ : for all $\left.a \in A\right\}$ which is induced from $\left\{\Theta_{a}\right.$ : for all $\left.a \in A\right\}$.

In [65], Wang et al. employed down-sets and up-sets to study covering-based rough sets. They used them to characterize reducible elements and minimal descriptions, which are 
quite important concepts in covering-based rough sets. They also gave lattice structure and topology in covering-based rough sets using down-sets and up-sets. For the theory of lattices, we refer to [53].

Definition 3.18. Let $(U, \prec)$ be a poset. For $X \subseteq U$, define $\downarrow X=\{x \in U$ : there exists $a \in X, x \prec a\}$. Then $\downarrow X$ is called a down-set of $X$ on the poset $(U, \prec)$.

Definition 3.19. Let $(U, \prec)$ be a poset. For $X \subseteq U$, define $\uparrow X=\{x \in U$ : there exists $a \in X, a \prec x\}$. Then $\uparrow X$ is called a up-set of $X$ on the poset $(U, \prec)$.

The reducible element (see Definition 3.10) and minimal description (see Definition 3.14) in $\mathcal{C}$ can be characterized through down-sets by the following result:

Proposition 3.20. Let $\mathcal{C}$ be a covering of a non-empty set $U$. Then $(\mathcal{C}, \subseteq)$ is a poset. Suppose $K \in \mathcal{C}$. Then $K$ is reducible in $\mathcal{C}$ if and only if $K=\bigcup(\downarrow\{K\}-\{K\})$ and $M d(x)=\{K \in \mathcal{C}: x \in K$ and $\downarrow\{K\}=\{K\}\}$, for all $x \in U$.

Further, the authors proposed the lattice and topological structures on coverings as follows:

Definition 3.21. Let $\mathcal{C}$ be a covering of a non-empty set $U$. Define $\tau_{*}=\left\{\mathcal{C}^{\prime} \subseteq \mathcal{C}: \downarrow \mathcal{C}^{\prime}=\mathcal{C}^{\prime}\right\}$ and $\tau^{*}=\left\{\mathcal{C}^{\prime} \subseteq \mathcal{C}: \uparrow \mathcal{C}^{\prime}=\mathcal{C}^{\prime}\right\}$.

Proposition 3.22. $\quad$ (1) Let $\mathcal{C}$ be a covering of a non-empty set $U$. Then $\left(\tau_{*}, \cup, \cap\right)$ and $\left(\tau^{*}, \cup, \cap\right)$ are lattices.

(2) Let $\mathcal{C}$ be a covering of $U$. Then $\left(\mathcal{C}, \tau_{*}\right)$ and $\left(\mathcal{C}, \tau^{*}\right)$ are topological spaces. Further, $\beta_{*}=\{\downarrow\{K\}: K \in \mathcal{C}\}$ and $\beta^{*}=\{\uparrow\{K\}: K \in \mathcal{C}\}$ are topological bases of the topological spaces $\left(\mathcal{C}, \tau_{*}\right)$ and $\left(\mathcal{C}, \tau^{*}\right)$, respectively.

Apart from these studies, Medhat in [34] showed that a cover $\mathcal{C}$ forms as a subbase for a topology on $U$. The lower approximation generated by the cover $\mathcal{C}$ is the interior of a set defined by this topology while the upper approximation may not contain the closure of a set with respect to this topology.

In [54], Restrepo et al. studied relationships between approximation operators defined from neighborhood operators and the approximation operators in the covering based rough set theory. Note that a complete lattice $(L, \leqslant)$ is a lattice which has least upper bound and greatest lower bound for every subset $M$ of $L$ in $(L, \leqslant)$.

Definition 3.23. Let $f$ and $g$ be two self-maps on a complete Boolean lattice $B$. We say that $g$ is the dual of $f$, if for all $x \in B, g\left(x^{c}\right)=(f(x))^{c}$, where $x^{c}$ represents the complement of $x \in B$.

Definition 3.24. Let $f$ and $g$ be two self-maps on a complete Boolean lattice $B$. We say that $g$ is a conjugate of $f$, if for all $x, y \in B, x \wedge f(y)=0$ if and only if $y \wedge g(x)=0$. If $g$ is a conjugate of $f$, then $f$ is a conjugate of $g$. If a map $f$ is the conjugate of itself, then $f$ is called self-conjugate.

Definition 3.25. A neighborhood operator is a mapping $N: U \rightarrow \mathcal{P}(U)$. If $N(x) \neq \emptyset$ for all $x \in U, N$ is called a serial neighborhood operator. If $x \in N(x)$ for all $x \in U, N$ is called a reflexive neighborhood operator.

The authors defined approximation operators using a neighborhood operator. Let $N$ be a neighborhood operator. Then the authors defined two lower approximations $L_{1}^{N}$ and $L_{2}^{N}$ as follows:

$L_{1}^{N}(A)=\{x: N(x) \subseteq A\}$,

$L_{2}^{N}(A)=\bigcup\{N(x): N(x) \subseteq A\}$, 
and three upper approximations $G_{1}^{N}, G_{2}^{N}$ and $G_{3}^{N}$ as follows:

$G_{1}^{N}(A)=\bigcup\{N(x): x \in A\}$

$G_{2}^{N}(A)=\{x \in U: N(x) \cap A \neq \emptyset\}$

$G_{3}^{N}(A)=\bigcup\{N(x): N(x) \cap A \neq \emptyset\}$.

Then $G_{1}^{N}$ is conjugate of $G_{2}^{N}$; the pair $\left(G_{2}^{N}, L_{1}^{N}\right)$ are conjugate if and only if $G_{1}^{N}=G_{2}^{N}$; and $L_{2}^{N}$ is dual of $G_{2}^{N}$. The following proposition is the characterization of pairs of dual approximation operators based on neighborhoods.

Proposition 3.26. Let $N$ be a neighborhood operator. The following statements are equivalent:

(i) For all $x, y \in U, N$ satisfies $y \in N(x) \Rightarrow x \in N(y)$.

(ii) $G_{2}^{N}=G_{3}^{N}$.

(iii) There exists a symmetric binary relation $\Theta$ on $U$ such that $N(x)=\{y \in U: x \Theta y\}$.

Further, we can construct approximation spaces on a subset of $U$. This was done in [8], where two subsystems (i.e., family of subsets of subsets of $U$ ) were constructed. As there was no connection between two subsystems, the corresponding lower approximation and upper approximation were not dual to each other. This problem was resolved by $\mathrm{Li}$ and Jing in [28]. Li and Jing chose a subsystem $\mathcal{S}$ and its dual subsystem $\mathcal{S}^{c}=\left\{A^{c}: A \in \mathcal{S}\right\}$ to construct a general approximation space.

Definition 3.27. Let $U$ be a non-empty set and $\mathcal{S}$ be a family of subsets of $U$. Then $\left(U, \mathcal{S}, \mathcal{S}^{c}\right)$ is called a general granular space. For any $X \subseteq U$, the lower and upper approximations of $X$ in $\left(U, \mathcal{S}, \mathcal{S}^{c}\right)$ are defined, respectively, by

$X_{*}=\underline{\mathcal{S}}(X)=\bigcup\{A \in \mathcal{S}: A \subseteq X\}$,

$X^{*}=\overline{\mathcal{S}}(X)=\bigcap\left\{A \in \mathcal{S}^{c}: X \subseteq A\right\}$.

The Operator $\underline{\mathcal{S}}$ satisfies the properties of an interior operator except $\underline{\mathcal{S}}(U)=U$ and $\underline{\mathcal{S}}(X \cap Y)=\underline{\mathcal{S}}(X) \cap \underline{\mathcal{S}}(Y)$. Similarly $\overline{\mathcal{S}}$ satisfies the properties of a closure operator except $\overline{\mathcal{S}}(\emptyset)=\emptyset$ and $\overline{\mathcal{S}}(X \cup Y)=\overline{\mathcal{S}}(X) \cup \overline{\mathcal{S}}(Y)$.

The new model can be considered as an extension of the rough set models on covering approximation spaces, knowledge spaces, topological spaces and Pawlak's approximation spaces by the following results:

(1) Let $\left(U, \mathcal{S}, \mathcal{S}^{c}\right)$ be a general granular space. Then $\mathcal{S}$ is a covering of $U$, if and only if $\underline{\mathcal{S}}(U)=U$ or $\overline{\mathcal{S}}(\emptyset)=\emptyset$.

(2) Let $\left(U, \mathcal{S}, \mathcal{S}^{c}\right)$ be a general granular space. Then $\mathcal{S}$ is a knowledge space on $U$, or $\mathcal{S}^{c}$ is a closure system on $U$, if and only if $\underline{\mathcal{S}}(\mathcal{S}) \subseteq \mathcal{S}$ or $\overline{\mathcal{S}}\left(\mathcal{S}^{c}\right) \subseteq \mathcal{S}^{c}$.

(3) Let $\left(U, \mathcal{S}, \mathcal{S}^{c}\right)$ be a general granular space, where $\mathcal{S}$ is a covering of $U$. Then $\mathcal{S}$ is a base for a topology on $U$, if and only if for any $X_{1}, X_{2} \in \mathcal{P}(U), \underline{\mathcal{S}}\left(X_{1} \cap X_{2}\right)=$ $\underline{\mathcal{S}}\left(X_{1}\right) \cap \underline{\mathcal{S}}\left(X_{2}\right)$ or $\overline{\mathcal{S}}\left(X_{1} \cup X_{2}\right)=\overline{\mathcal{S}}\left(X_{1}\right) \cup \overline{\mathcal{S}}\left(X_{2}\right)$.

(4) Let $\left(U, \mathcal{S}, \mathcal{S}^{c}\right)$ be a general granular space. Then there is a subsystem $\mathcal{D} \subseteq \mathcal{S}$ such that $\mathcal{D}$ is a partition of $U$ and $\underline{\mathcal{D}}=\underline{\mathcal{S}}$. Further $\overline{\mathcal{D}}=\overline{\mathcal{S}}$, if and only if $\underline{\mathcal{S}}$ satisfies $\underline{\mathcal{S}}(U)=U, \underline{\mathcal{S}}(X \cap Y)=\underline{\mathcal{S}}(X) \cap \underline{\mathcal{S}}(Y)$ and $\overline{\mathcal{S}}(\underline{\mathcal{S}}(X)) \subseteq X$, or $\overline{\mathcal{S}}$ satisfies $\overline{\overline{\mathcal{S}}}(\emptyset)=\emptyset$, $\overline{\mathcal{S}}(X \cup Y)=\overline{\mathcal{S}}(X) \cup \overline{\mathcal{S}}(Y)$ and $X \subseteq \underline{\mathcal{S}}(\overline{\mathcal{S}}(X))$.

\subsection{Topological aspects of logic and algebra on rough sets}

It is hard to authenticate who was the first to use the algebraic methods. The studies in the logic of Boole himself led to the notion which nowadays we call Boolean algebra [53]. Tarski [58] introduce the turning point in the algebraic study of logic as the method of treating formulae, or equivalence classes of formulae as elements of abstract algebra, which is now known as the Lindenbaum-Tarski algebra. The primary use of the LindenbaumTarski algebra is to study the correspondence between logic and abstract algebras. Of course, the algebra corresponding to classical logic is a Boolean algebra. Boolean and 
other algebras are somewhat general structures. A proper representation theorem establishes their deeper mathematical meaning. The representation theorem, establishes a relationship between logic, algebra, set theory, and topology [57]. The first representation theorem for Boolean algebras was established by Stone (in [57]) and stated the following:

Theorem 3.28. Every Boolean algebra $A$ is isomorphic to a field of sets. More exactly, $A$ is isomorphic to a field of subsets of its Stone space $S$, which is a compact and totally disconnected Hausdorff space.

Many researchers have contributed to this area. Here, we discuss some important researchers of topology done in the field of logic and algebra of classical rough sets. Wasilewska [66] studied the idea of rough equality of sets which leads to a definition of new classes of algebras, called topological quasi-Boolean algebras and topological rough algebras. Rough algebra, introduced by Banerjee and Chakraborty [2], is a particular case of Boolean algebra, which is a slight abstraction of De-Morgan's lattices. The authors also proved the representation theorem for topological rough algebras:

For every topological quasi-Boolean algebra (topological rough algebra) there exists a monomorphism from topological quasi-Boolean algebra into a topological quasi-field (roughfield) of sets of a topological space. A rough algebra is isomorphic to a topological roughfield of sets.

In [75], Yao compared the constructive and algebraic approaches in the study of rough sets. Different classes of rough set algebras are obtained from different types of binary relations. Using constructive approach, a rough set algebra can be defined as follows:

Definition 3.29. Let $(U, \Theta)$ be a Yao's approximation space, where $\Theta$ is a binary relation on a non-empty set $U$. The pair of approximation operators is defined as, $X^{*}=\left\{x: \Theta_{s}(x) \subseteq X\right\}, X_{*}=\left\{x: \Theta_{s}(x) \cap X \neq \emptyset\right\}$, where $\Theta_{s}(x)=\{y: x \Theta y\}$.

Then the system $(\mathcal{P}(U), \cap, \cup, c, *)$ is called a rough set algebra, where $\cap, \cup$, and $c$ are set intersection, union and complement, respectively, and $*$ denotes the pair of approximation operators.

In the algebraic approach, one defines a pair of dual approximation operators and states axioms that must be satisfied by the operators. In an algebraic or axiomatic approach, the primitive notion is a system $(\mathcal{P}(U), \cap, \cup, c, \mathbf{L}, \mathbf{H})$, where $(\mathcal{P}(U), \cap, \cup, c)$ is the set algebra, and $\mathbf{L}, \mathbf{H}: \mathcal{P}(U) \rightarrow \mathcal{P}(U)$ are unary operators on the power set $\mathcal{P}(U)$. We call $\mathbf{L}$ and $\mathbf{H}$ the approximation operators, indicating their intended physical interpretation. They are defined by axioms without direct reference to the binary relation.

Definition 3.30. Let $\mathbf{L}, \mathbf{H}: \mathcal{P}(U) \rightarrow \mathcal{P}(U)$ be two unary operators on the power set $\mathcal{P}(U)$. They are dual operators if

(i) $\mathbf{L}(X)=\left(\mathbf{H}\left(X^{c}\right)\right)^{c}$,

(ii) $\mathbf{H}(X)=\left(\mathbf{L}\left(X^{c}\right)\right)^{c}$,

for all $X \subseteq U$.

For the algebraic study of the theory of rough sets, the following theorem can be easily obtained from the constructive approach.

Theorem 3.31. Suppose $\boldsymbol{L}, \boldsymbol{H}: \mathcal{P}(U) \rightarrow \mathcal{P}(U)$ are dual unary operators. There exists a binary relation $\Theta$ on $U$ such that $\boldsymbol{L}(X)=X_{*}$ and $\boldsymbol{H}(X)=X^{*}$ for all $X \subseteq U$, if and only if $\boldsymbol{L}$ and $\boldsymbol{H}$ satisfy the properties:

(i) $\boldsymbol{L}(U)=U$,

(ii) $\boldsymbol{L}(X \cap Y)=\boldsymbol{L}(X) \cap \boldsymbol{L}(Y)$,

(iii) $\boldsymbol{H}(\emptyset)=\emptyset$,

(iv) $\boldsymbol{H}(X \cup Y)=\boldsymbol{H}(X) \cup \boldsymbol{H}(Y)$.

A counterpart of Definition 3.30 is therefore obtained in the algebraic framework. 
Definition 3.32. Let $\mathbf{L}, \mathbf{H}: \mathcal{P}(U) \rightarrow \mathcal{P}(U)$ be a pair of dual operators. If $\mathbf{L}$ satisfies axioms $(i)$ and $(i i)$, or equivalently, $\mathbf{H}$ satisfies axioms (iii) and $(i v)$ of above theorem, then the system $(\mathcal{P}(U), \cap, \cup, c, \mathbf{L}, \mathbf{H})$ is called a rough set algebra, and $\mathbf{L}$ and $\mathbf{H}$ are called approximation operators.

Set-theoretic operators $\mathbf{L}$ and $\mathbf{H}$ are viewed as the counterparts of the necessity and possibility operators in modal logic [77]. The algebraic approach is more flexible and general than the constructive approach.

In [10], Eklund et al. showed that a partially ordered monad [32] contains sufficient structure for modeling monadic topologies, rough sets, and Kleene algebras. The authors showed how monads $[3,32]$ could be used to generalize and interpret rough situations. In particular, a partially ordered ordinary power set monad contains sufficient structure to provide rough set operations.

Järvinen et al. [18-21] studied lattice structure of rough approximations and rough sets determined by indiscernibility relations which are not necessarily reflexive, symmetric or transitive.

Definition 3.33. Let $P$ and $Q$ be two preorders (reflexive and transitive relations). A pair $(f, g)$ of maps $f: P \rightarrow Q$ and $g: Q \rightarrow P$ is called a Galois connection if, $f(p) \leq q$ if and only if $p \leq g(q)$. The map $g$ is called the adjoint of $f$. The map $f$ is called the co-adjoint of $g$.

Any map $f$ between two complete lattices has an adjoint $g$, and the pair $(f, g)$ is a Galois connection, if and only if $f$ is a complete join-morphism.

The upper approximation operator determined by an arbitrary binary relation $\Theta$ on a universe $U$ is a complete join-morphism on the power set of $U$. Therefore it induces a Galois connection such that the adjoint is the lower approximation operator determined by the inverse relation of $\Theta$. The main properties of rough approximation operators follow from the conventional and well-known properties of Galois connections on Boolean lattices. The set of fixed points of the upper approximation operation determined by a reflexive indiscernibility relation $\Theta$ forms an Alexandrov topology $\tau_{\Theta}$, and if the relation $\Theta$ is also symmetric, the topology $\tau_{\Theta}$ is closed under complementation. Moreover, if the underline relation $\Theta$ is reflexive, then the upper approximation operator serves as the smallest neighborhood operator of the Alexandrov topology $\tau_{\Theta}$.

Some widely known and well established topological notions are closely related to notions and results introduced and rediscovered in the rough set literature. These were studied by Vlach [64] where he discussed the notion of an approximation space through a pair of isotonic function [14], and then compared it with Pawlak's approximation space. The author showed that topologies induced by approximation operators of the rough set theory are uniform topologies (see $[16,37]$ ).

Let $R$ be an equivalence relation in $U$ and let $D_{R}$ be the partition of $U$ induced by $R$. A subset $X$ of $U$ is $R$-definable if it is either empty or a member of $D_{R}$ or union of two or more members of $D_{R}$. Alternatively, the lower approximations of subsets of $U$ are values of the set-to-set function $f_{R}: \mathcal{P}(U) \rightarrow \mathcal{P}(U)$ defined by

$$
f_{R}(X)=\bigcup\left\{A \in D_{R}: A \subseteq X\right\}, X \subseteq U .
$$

It can easily be verified that, the dual of $f_{R}$ is

$$
f_{R}^{d}(X)=\bigcup\left\{A \in D_{R}: A \cap X \neq \emptyset\right\} .
$$

Hence $\left(U,\left(f_{R}, f_{R}^{d}\right)\right)$ forms an approximation space where $f_{R}(X)$ and $f_{R}^{d}(X)$ are the lower and upper approximations respectively. The upper approximation of a subset of $U$ are values of the function that is dual to the function whose values are lower approximation of the given subset of $U$, and vice versa. It can easily be seen that, for all subsets $X$ and $Y$ of $U$, we have

(1) $f_{R}(X) \subseteq X \subseteq f_{R}^{d}(X)$, i.e., $\left(U,\left(f_{R}, f_{R}^{d}\right)\right)$ is a uniform approximation space. 
(2) $X \subseteq Y$ implies $f_{R}(X) \subseteq f_{R}(Y)$ and $f_{R}^{d}(X) \subseteq f_{R}^{d}(Y)$, i.e., $f_{R}$ and $f_{R}^{d}$ are isotones.

(3) $f_{R}\left(f_{R}(X)\right)=f_{R}(X)$ and $f_{R}^{d}\left(f_{R}^{d}(X)\right)=f_{R}^{d}(X)$.

(4) $f_{R}\left(f_{R}(X)\right)=f_{R}^{d}\left(f_{R}(X)\right)$.

(5) $f_{R}\left(f_{R}^{d}(X)\right)=f_{R}^{d}\left(f_{R}^{d}(X)\right)$.

Thus, $f_{R}$ is idempotent and contractive and $f_{R}^{d}$ is a closure function, i.e., $f_{R}^{d}$ is idempotent and expansive. Also it is clear that, Pawlak's $R$-definable sets are exact in the approximation space $\left(U,\left(f_{R}, f_{R}^{d}\right)\right)$. Pawlak's lower and upper approximations of subsets of $U$ are $R$-definable sets. It is also useful to notice that, for all $X$ and $Y$,

$f_{R}(X \cap Y)=f_{R}(X) \cap f_{R}(Y)$

$f_{R}^{d}(X \cup Y)=f_{R}^{d}(X) \cup f_{R}^{d}(Y)$,

$f_{R}(\emptyset)=f_{R}^{d}(\emptyset)=\emptyset$ and $f_{R}(U)=f_{R}^{d}(U)=U$.

We know that $\mathcal{P}(U)$ is a complete lattice. The set of all functions which map the power set $\mathcal{P}(U)$ of a non-empty set $U$ into itself, inherits from $\mathcal{P}(U)$ a set algebra and inclusion. Moreover, this set is also a semigroup with respect to the composition of functions and the identity function. Since Pawlak's rough sets can be represented by ordered pairs $\left(f_{R}(X), f_{R}^{d}(X)\right)$ of lower and upper approximations, therefore the relation ' $\leq$ ' gives a natural partial order ' $\preceq$ ' on the collection of rough sets in $(U, R)$ defined as $\left(f_{R}(X), f_{R}^{d}(X)\right) \preceq\left(f_{R}(Y), f_{R}^{d}(Y)\right)$. This partially ordered set is a complete Stone lattice [5]. The ordered set of rough sets determined analogously by relations that are simultaneously symmetric and transitive but not necessarily reflexive also forms a complete Stone lattice (see [19]). However, Järvinen [17,19] showed that the ordered set of rough sets determined by tolerance relations and transitive relations are not even semilattices necessarily, in general.

\subsection{Theories related to rough sets}

In this section, we give a brief survey on some of the theories related to classical rough sets and topology and their conjoint applications.

3.5.1. Near set theory. In $[44,45]$, Peters et al. discussed how to describe and compare visual objects. In the process, they investigated the conjoint study of rough sets and proximity spaces $[23,24,52,61-63]$. The authors considered two forms of nearness relations, namely, a spatial and a descriptive relation to study nearness of objects. There is a natural transition from the two forms of nearness relations to nearness in a generalized approximation space. Let $\delta \subseteq \mathcal{P}(U) \times \mathcal{P}(U)$ denote a nearness (proximity) relation on a non-empty set $U$. For $B, C \subseteq U$, we write $B \delta C$ ( $B$ is spatially near $C$ ). If $B$ is not near (far) $C$ (denoted by $B \bar{\delta} C$, where $\bar{\delta}=\mathcal{P}(U) \times \mathcal{P}(U)-\delta$ ), then $B \cap C=\emptyset$. Now let us define the two nearness relations given in the paper.

Definition 3.34. [36] The relation $\delta \subseteq \mathcal{P}(U) \times \mathcal{P}(U)$ is an Efremovič proximity (also called an EF-proximity), if and only if, for $A, B, C \in \mathcal{P}(U)$, the following axioms hold:

(1) $A \delta B \Rightarrow A$ and $B$ are not empty,

(2) $A \cap B \neq \emptyset \Rightarrow A \delta B$,

(3) $A \delta B \Rightarrow B \delta A$ (symmetry),

(4) $A \delta(B \cup C)$ if and only if $A \delta B$ or $A \delta C$,

(5) Efremovič axiom: $A \bar{\delta} B \Rightarrow A \bar{\delta} C$ and $B \bar{\delta} C^{c}$, for some $C \subseteq U$.

The pair $(U, \delta)$ is called an EF-proximity space.

Let $U$ be a non-empty set and let $\Phi=\left\{\phi_{1}, \ldots, \phi_{i}, \ldots, \phi_{n}\right\}$ be a set of probe functions, i.e., $\phi_{i}: U \rightarrow \mathbb{R}$ represents features of each $x$. Let $\Phi(x)$ denote a feature vector for the object $x$, or a vector of feature values that describe $x$, i.e., $\Phi(x)=\left(\phi_{1}(x), \ldots, \phi_{i}(x), \ldots, \phi_{n}(x)\right)$. A feature vector provides a description of an object.

Define an equivalence relation $R_{\Phi} \subseteq U \times U$ by, $R_{\Phi}=\{(x, y) \in U \times U: \Phi(x)=\Phi(y)\}$. The 
relation $R_{\Phi}$ is called an $\Phi$-indiscernibility relation [42] and the pair $\left(U, R_{\Phi}\right)$ is a Pawlak's approximation space. The upper approximation of $E \subseteq U$ is defined by,

$$
E^{*}=\bigcup_{[x]_{\Phi} \cap E \neq \emptyset}[x]_{\Phi}=\bigcup_{x \in U: \Phi(x) \in \mathcal{Q}_{\Phi}(E)}[x]_{\Phi},
$$

where $\mathcal{Q}_{\Phi}(E)=\{\Phi(x): x \in E\}$ is called the description of $E$ relative to $\Phi$ and $[x]_{\Phi}$ is the equivalence class of $x \in U$ determined by $R_{\Phi}$. The lower approximation of $E \subseteq U$ is defined by,

$$
E_{*}=\bigcup_{[x]_{\Phi} \subseteq E}[x]_{\Phi}=\bigcup_{x \in U: \Phi(x) \in \mathcal{Q}_{\Phi}(E)^{+}}[x]_{\Phi},
$$

where $\mathcal{Q}_{\Phi}(E)^{+}=\left\{v \in Q_{\Phi}(E)\right.$ : for all $\left.x \in E, \Phi(x)=v\right\}$.

Now define the descriptive intersection $\cap_{\Phi}$ of subsets $A$ and $B$ of $X$ by

$$
A \cap_{\Phi} B=\left\{x \in U: \Phi(x) \in \mathcal{Q}_{\Phi}(A) \cap \mathcal{Q}_{\Phi}(B)\right\} .
$$

Then $A \cap_{\Phi} B=A^{*} \cap B^{*}$. For any two non-empty sets $A$ and $B$, descriptive union is defined by

$$
A \cup_{\Phi} B=\left\{x \in U: \Phi(x) \in \mathcal{Q}_{\Phi}(A) \cup \mathcal{Q}_{\Phi}(B)\right\} .
$$

We have $A \cup_{\Phi} B=(A \cup B)^{*}$. Let us assume that $\Phi(x) \in \mathbb{R}^{n}$, where $\mathbb{R}$ is the set of real numbers. The descriptive proximity relative to $\Phi$ is defined as follows:

Definition 3.35. [45] The binary relation $\delta_{\Phi}$ is a descriptive Efremovič-proximity (EFproximity), provided the following axioms are satisfied for $A, B, C \subseteq U$ :

(i) $A \delta_{\Phi} B \Rightarrow A \neq \emptyset, B \neq \emptyset$,

(ii) $A \cap_{\Phi} B \neq \emptyset \Rightarrow A \delta_{\Phi} B$,

(iii) $A \delta_{\Phi} B \Rightarrow B \delta_{\Phi} A$,

(iv) $A \delta_{\Phi}(B \cup C) \Leftrightarrow A \delta_{\Phi} B$ or $A \delta_{\Phi} C$,

(v) $A \bar{\delta}_{\Phi} B \Rightarrow A \bar{\delta}_{\Phi} C$ and $B \bar{\delta}_{\Phi} C^{c}$, for some $C \subseteq U$.

The pair $\left(U, \delta_{\Phi}\right)$ is called a descriptive EF-proximity space (briefly, descriptive EF-space). Two points are descriptively distinct if they have different descriptions. For distinct points $x, y \in U$, a descriptive EF-proximity is separated, if and only if, it satisfies

(vi) $\{x\} \delta_{\Phi}\{y\} \Rightarrow \Phi(x)=\Phi(y)$ (c.f. EF-Proximity Separation Axiom [36]).

The tuple $\left(U, R_{\Phi}, \delta_{\Phi}\right)$ denotes a descriptive nearness approximation space, which is a Pawlak's approximation space endowed with the descriptive EF-proximity relation $\delta_{\Phi}$.

Let $\left(U, R_{\Phi}, \delta_{\Phi}\right)$ be a descriptive nearness approximation space and let $A, B \subseteq U$. Then, for descriptively near sets, the following statements are equivalent:

(a) $A \delta_{\Phi} E$,

(b) there exists $x \in U$ such that $[x]_{\Phi} \cap A \neq \emptyset$ and $[x]_{\Phi} \cap E \neq \emptyset$,

(c) $A^{*} \cap E^{*} \neq \emptyset$,

(d) there exists $y \in A$ and $z \in E$ such that $[y]_{\Phi}=[z]_{\Phi}$.

Concepts of nearness and remoteness of granules are defined using descriptive nearness.

Consider the following example: Let $\left(U, R_{\Phi}, \delta_{\Phi}\right)$ be the descriptive nearness approximation space, where $R_{\Phi}=\{(x, y): \Phi(x)=\Phi(y)\}$ shown in Figure 1(A). Here $U$ is a set of picture elements (pixels) in a micro-fossil image as shown in Figure 1(B) and $\Phi$ is the set of probe functions representing color and greyscale intensity features of pixels in $U$. In this example, the authors compared the age groups of different micro-fossils using nearness theory on rough sets of micro-fossils. Let the distinguished collection of subsets $\mathcal{B} \subset \mathcal{P}^{2}(U)$ in Figure $1(\mathrm{~B})$ be $\mathcal{B}=\left\{B_{1}, B_{2}, E_{1}, E_{2}, E_{3}, E_{4}\right\}$. Observe that the set $A$ in Figure $1(\mathrm{~B})$ and each of the subsets in $\mathcal{B}$ is a rough set because the lower approximation of each subset is empty. These selected subsets are of interest because they contain parts of the bodies for micro-fossils of different age groups. The bodies have varying mass, depending on the age of the micro-fossils. To extract an upper approximation nearness granule from the distinguished subsets, we consider portions of the upper approximation of $A$ relative to 


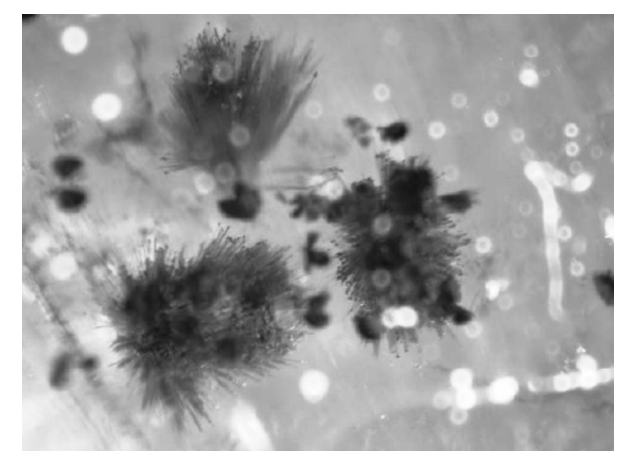

(a) Complex Fossil Image

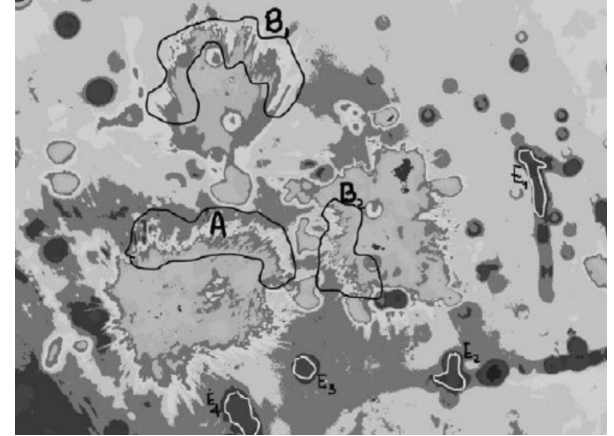

(b) Sample Rough Set-Based Setae Granules

Figure 1. Microfossil Image

the upper approximation of each subset in $\mathcal{B}$ and portions of the upper approximation of each subset in $\mathcal{B}$ relative to the upper approximation of $A$.

$$
\begin{gathered}
\left.\operatorname{Dis}_{\Phi^{*}}(A, C)=\Phi^{*} A-\Phi^{*} C \text { (portion of } \Phi^{*} A \text { different from } \Phi^{*} C\right), \\
\left.\operatorname{Ind}_{\Phi^{*}}(A, C)=\Phi^{*} A \cap \Phi^{*} C \text { (portion of } \Phi^{*} A \text { common with } \Phi^{*} C\right), \\
\left.\operatorname{Dis}_{\Phi^{*}}(C, A)=\Phi^{*} C-\Phi^{*} A \text { (portion of } \Phi^{*} C \text { different from } \Phi^{*} A\right), \\
\text { where } C \in\left\{B_{1}, B_{2}, E_{1}, E_{2}, E_{3}, E_{4}\right\} .
\end{gathered}
$$

We are only interested in comparing the descriptions of pixels in $A^{*}$ with descriptions of pixels in each upper approximation of distinguished subsets from $\mathcal{B}$. Obviously, from the above definition $\operatorname{Ind}_{\Phi^{*}}(A, C) \neq \emptyset$, i.e., $A$ and $C$ are near. However, information encoded in $\operatorname{Dis}_{\Phi^{*}}(A, C)$ and $D i s_{\Phi^{*}}(C, A)$ is also useful when one would like to compare the sets $A, C$. For example, the sets $A, C$ may be near but they differ as described by $\operatorname{Dis}_{\Phi^{*}}(A, C)$ and $\operatorname{Dis}_{\Phi^{*}}(C, A)$. The proposed approach of extracting a nearness granule is motivated by an interest in comparing the pixels in portions of the upper approximations of the subsets in $B$ with the upper approximation of $A$.

The beauty of this approach is that we can utilize our knowledge of the equivalence classes in $U$ to learn to what extent the classes represented by $A^{*}$ are also represented to a greater or lesser degree in the upper approximation of one or more of the distinguished subsets in $B$, and vice versa. With this approach in mind, we obtain a sample upper approximation nearness granule, namely,

$$
\begin{gathered}
A^{*}=\operatorname{Dis}_{\Phi^{*}}(A, C), \text { where } C \in\left\{E_{1}, E_{2}, E_{3}, E_{4}\right\}, \\
B_{i}^{*}=D_{i s \Phi^{*}}\left(B_{i}, E_{j}\right)^{*}, \text { where } i \in\{1,2\} \text { and } j \in\{1,2,3,4\} .
\end{gathered}
$$

For example, $A^{*} \delta_{\Phi} B_{1}^{*}$ ( $A^{*}$ is descriptively near $B_{1}^{*}$ ), since a portion of the upper approximation containing purple pixels in the equivalence class (for setae) is represented by $A^{*}$ and $B_{1}^{*}$.

3.5.2. Finite functional topology, Discrete dynamical system, and Rough set theory. Functional information system provides a detailed information for a specific type of activity or related group of activities, as well as summarized information for management control of such activities. Here, we will discuss the contribution of Wolski [69] in this field. Wolski described discrete dynamical system through the concept of rough sets for the study of finite functional topology. The author focused on functional system which describe some process represented by a function $\rho$. In this paper, he used the topological structure generated by lower approximations of subsets of $U$, and named it as approximation topological space. For a topological space $(U, \tau)$, the relation of set inclusion on $\tau$ can be converted into a preorder $\preceq$ defined on elements of $U$, called the specialization preorder, as follows: 


$$
a \preceq b \text { if and only if } \operatorname{cl}(\{a\}) \subseteq \operatorname{cl}(\{b\}),
$$

where $\operatorname{cl}(\{a\})$ is the closure of $\{a\}$ with respect to the topological space $(U, \tau)$. For an arbitrary preordered set $(U, \preceq)$, there is always a topology $\tau$ whose specialization preorder is $\preceq$.

Definition 3.36. Let $(U, \preceq)$ be a preordered set. A specialization topology on $U$ is a topology $\tau$ with a specialization preorder $\preceq$ such that every automorphism of $U$ is a homeomorphism of $(U, \tau)$.

As there exists a one-to-one correspondence between Alexandrov topologies on a set $U$ and preorders on $U$, so for a preordered set $(U, \preceq)$, we shall denote the corresponding Alexandrov topological space by $\left(U, \tau_{\preceq}\right)$. Thus for a given information system $S=$ $(U, \mathcal{A}, V, f)$ equipped with its information order $\preceq,(U, \tau \preceq)$ is an Alexandrov topological space. If $S=(U, \mathcal{A}, V, f)$ is a complete information system having information order $\preceq$ (which is an equivalence relation), then $\left(U, \tau_{\preceq}\right)$ is a completely regular Alexandrov topological space $[1,22]$. Since the author started with the concept of an information system, his interest was restricted to discrete dynamical systems. These systems have been already investigated in the context of computer science in $[12,35]$.

Definition 3.37. A discrete dynamical system is a pair $(U, \rho)$ where $U$ is a set and $\rho: U \rightarrow U$ is simply a function from $U$ into itself.

A key concept in the study of discrete dynamical systems is the orbit of a point.

Definition 3.38. Let $\rho: U \rightarrow U$. The $\rho$-orbit of $a \in U$, often called the $\rho$-trajectory of $a$, $\mathcal{O}_{\rho}(a)$, is defined as a sequence:

$$
\left(\rho^{0}(a), \rho^{1}(a), \rho^{2}(a), \rho^{3}(a), \ldots\right),
$$

where $\rho^{k}=\rho o \rho^{k-1}$. The usual interpretation of orbit is that the iterations of $\rho^{n}(a)$ describe the evolution of $a$ in discrete time $n$. Of course, any orbit $\mathcal{O}_{\rho}(a)$ can be converted into a set $\mathcal{O}_{s \rho}(a)$ :

$$
\mathcal{O}_{s \rho}(a)=\left\{b \in U: b=\rho^{n}(a), \text { for some } n\right\},
$$

$a \in U$. The $\rho$-orbit of a is cyclic if $\rho^{n}(a)=a$, for some $n \geq 1$. We also say that $a$ is a cyclic point (or a periodic point) for $\rho$. The $\rho$-orbit of a is called eventually cyclic if $\rho^{n}(a)=\rho^{m}(a)$, for some $n, m$ with $n \neq m$, and in this case $a$ is eventually cyclic point for $\rho$.

Let $U$ be a set and $\rho: U \rightarrow U$ be a function. Define

$$
\tau_{\rho}=\{A \subseteq U: \rho(A) \subseteq A\} \text {, where } \rho(A)=\{\rho(x): x \in A\} .
$$

It is easy to observe that $\tau_{\rho}$ is a topology on $U$, called functional topology on $U$. Let $(U, \rho)$ be a discrete dynamical system. Then $\left(U, \tau_{\rho}\right)$ is an Alexandrov topological space.

Definition 3.39. Let $(U, \tau)$ be a topological space. Then we say that the topology $\tau$ is a functional topology if there is a map $\rho: U \rightarrow U$ such that $\tau=\tau_{\rho}$ for some functional topological space $\left(U, \tau_{\rho}\right)$.

Let $\left(U, \tau_{\rho}\right)$ be a functional topological space. Then $\mathcal{O}_{s \rho}(a)$ is the minimal open neighborhood of $a \in U$ and the set $\left\{\mathcal{O}_{s \rho}(a): a \in U\right\}$ forms a minimal basis of $\tau_{\rho}$.

Definition 3.40. Let $S=(U, \mathcal{A}, V, f)$ be an information system. Let $\preceq$ denotes its information order and $\tau \preceq$ is specialization topology with a specialization preorder $\preceq$. Then $S$ is called functional if the corresponding topological space $(U, \tau \preceq)$ is functional. If $\tau_{\rho}=\tau_{\preceq}$ for some $\rho: U \rightarrow U$, then we say that the information system $S=(U, \mathcal{A}, V, f)$ describes the process $\rho$. 
The lower and upper approximations can be defined by means of orbits as follows:

$$
\begin{gathered}
\underline{A}=\left\{a \in U: \mathcal{O}_{s \rho}(a) \subseteq A\right\}, \\
\bar{A}=\left\{a \in U: \mathcal{O}_{s \rho}(a) \cap A \neq \emptyset\right\},
\end{gathered}
$$

$A \subseteq U$. Also, every $a \in U$ is an eventually cyclic point for $\rho$. Lastly, the author discussed the granular approach via dynamical systems. For a functional information system $S=$ $(U, \mathcal{A}, V, f)$ and $a \in U, \mathcal{O}_{\rho}(a)$ represents the smallest information granule containing $a$.

Definition 3.41. Let $S=(U, \mathcal{A}, V, f)$ be a functional information system (for some $\rho$ ). A granular orbit $\mathcal{G O}_{\rho}(a)$ of $a$ is defined as a sequence:

$$
\left(\mathcal{O}_{s \rho}\left(\rho^{0}(a)\right), \mathcal{O}_{s \rho}\left(\rho^{1}(a)\right), \mathcal{O}_{s \rho}\left(\rho^{2}(a)\right), \ldots, \mathcal{O}_{s \rho}\left(\rho^{m}(a)\right)\right) .
$$

Since every point of $a \in U$ is eventually cyclic, the author proved the following result:

Proposition 3.42. Let $S=(U, \mathcal{A}, V, f)$ be a functional information system, for some $\rho$ and $a \in U$. Then for all $\rho$-periodic points $b, c \in \mathcal{G O}_{\rho}(a), \mathcal{O}_{s \rho}(b)=\mathcal{O}_{s \rho}(c)$.

With the help of granular view of orbits, one could obtain quite rich lattice structures such as De Morgan Algebra [53] or 2-valued Łukasiewicz algebra (see [46]).

3.5.3. Formal concept analysis. In [68], Wolski established some formal relationships between formal concept analysis [11] and rough set theory based on the theory of finite topological approximations. The composition of the derivation operators from the formal concept analysis and the upper and lower approximation operators of rough set theory naturally emerge from the specialization order. This is an indication of some relationship between the formal concept analysis and the rough set theory. The composition of the derivation operator of formal concept analysis is the opposite operator with respect to the specialization order to the upper approximation of the rough set theory. Thus the formal concept analysis and the rough set theory taken together brought a semantics to the tense logic S4.t [49]. Thus, a topological space and its finite approximation are indistinguishable by means of the standard temporal language, i.e., in the case of topological approximate reasoning, we should employ tense logic $S 4$.t interpreted by the rough set theory and the formal concept analysis instead of the unimodal systems with the rough set theory semantics.

\section{Conclusion}

Topology is a useful theoretic framework for the study of rough sets. Approximation operators in the rough set theory are topological operators. Hence, a conjoint investigation of the rough set theory and topology is required, which has been done by many researchers since inception. We have done a literature review of the rough set theory and some of its variants which are designed using different bases: equivalence relational, arbitrary binary relational, topological, covering based. The discussion in this article focus on the topological structures on rough sets and some applications of topological structures on rough sets. Topological aspects of logic and algebra on rough sets are also discussed. The connections between the dynamical system and the rough set theory, the near set theory and the rough set theory, and the formal concept analysis and the rough set theory are also reviewed in this paper.

There are still some open problems/areas which can be explained in the future research of topological aspects of rough sets. Some of them are:

(1) The structures of rough uniform spaces, rough function spaces, and rough geometrical topology are still unexplored. 
(2) In the information system $S=(U, \mathcal{A}, V, f)$, the function $f: U \times \mathcal{A} \rightarrow V$ is independent of time. If we consider a time-dependent function $f$, then the values of function may vary with time. As a result, the topological structure on $U$ may also differ from time to time. The study of topological structures by considering a time-dependent function $f$ is still an open problem in this area.

(3) Topological structures on a information system $S=(U, \mathcal{A}, V, f)$, where $U$ is effect algebra [9] are still open to be studied.

(4) The field of topological groups still needs to be explored in the context of approximation spaces.

(5) Extension problems of topology on the topological structures defined on various approximation spaces still need attention from the researchers.

We hope this review will give a cutting edge and a wide reference to the conjoint research of rough sets and topology.

Acknowledgment. The authors express their thanks to the reviewers for the valuable suggestions towards the improvement of the paper. One of the authors, Pankaj Kumar Singh, gratefully acknowledge the University Grants Commission, New Delhi, India, for providing Senior Research Fellowship, student ID. 201516NETJRF-10459-1, to pursue research work.

\section{References}

[1] F.G. Arenas, Alexandroff spaces, Acta Math. Univ. Comenian. 68, 17-25, 1999.

[2] M. Banerjee and M.K. Chakraborty, Rough Consequence and Rough Algebra, in: Rough Sets, Fuzzy Sets and Knowledge Discovery, Proceedings of the International Workshop on Procedings of Rough Sets and Knowledge Discovery, (RSKD'93), Banff, Alberta, Canada, 1993, W.P. Ziarko, (Ed.), Springer Verlag, London, 196-207, 1994.

[3] M. Barr and C. Wells, Toposes, Triples and Theories, Springer-Verlag, 1985.

[4] W. Bartol, J. Miro, K. Pioro, and F. Rossello, On the coverings by tolerance classes, Inform. Sci. 166 (1-4), 193-211, 2004.

[5] G. Birkhoff, Lattice theory, Amer. Math. Soc. Colloq. Publ. 25, 1973.

[6] R. Biswas, Rough metric spaces, Bull. Pour. Les. Sous. Ens. Flous. Appl.(France) 68, 21-32, 1996.

[7] J. Dai, S. Gao, and G. Zheng, Generalized rough set models determined by multiple neighborhoods generated from a similarity relation, Soft Comput. 13, 2081-2094, 2013.

[8] J.P. Doignon and J.C. Falmagne, Knowledge Spaces, Springer, Heidelberg, 1999.

[9] A. Dvurecenskij and S. Pulmannova, New trends in quantum structures, Springer Science and Business Media, 2013.

[10] P. Eklund, M.A. Galán, and W. Gahler, Partially ordered monads for monadic topologies, Rough Sets and Kleene Algebras, Elect. Notes Theoret. Comp. Sci. 225, 67-81, 2009.

[11] B. Ganter and R. Wille, Formal Concept Analysis: Mathematical Foundations, Springer Science and Business Media, 2012.

[12] F. Geerts and B. Kuijpers, Topological formulation of termination properties of iterates of functions, Inform. Process. Lett. 89 (1), 31-35, 2004.

[13] M.A. Hajri, K. Belaid, and L.J. Belaid, Scattered spaces, compactification and an application to image classification problem, Tatra Mt. Math. Publ. 66 (1), 1-12, 2016.

[14] P.C. Hammer, Extended topology: Structure of isotonic functions, J. Reine Angew. Math. 213, 174-186, 1964.

[15] T. Herawan, Roughness of sets involving dependency of attributes in information systems, Int J. Softw. Eng. Appl. 9 (7), 111-126, 2015. 
[16] J.R. Isbell, Uniform Space, in: Math. Surveys Monogr No. 12, Amer. Math. Soc., 1964.

[17] J. Järvinen, Approximations and rough sets based on tolerances, in: Lecture Notes in Comput. Sci. 2005, 182-189, Springer-Verlag, Heidelberg, 2001.

[18] J. Järvinen, On the structure of rough approximations, Fund. Inform. 53, 135-153, 2002.

[19] J. Järvinen, The ordered set of rough sets, in: Lecture Notes in Comput. Sci. 3066, 49-58, Springer-Verlag, Berlin, Heidelberg, 2004.

[20] J. Järvinen, Lattice Theory for Rough Sets, in: Transactions on Rough Sets VI, LNCS 4374, 400-498, 2007.,

[21] J. Järvinen, M. Kono, and J. Kortelainen, Modal-like operators in boolean algebras, Galois connections and fixed points, Fund. Inform. 76, 129-145, 2007.

[22] J.L. Kelley, General Topology, Van Nostrand, 1955.

[23] M. Khare and S. Tiwari, L-approach merotopies and their categorical perspective, Demonstr. Math. XLV (3), 2012.

[24] M. Khare and S. Tiwari, Completion in a common supercategory of Met, UAP, wsAP and Near, Demonstr. Math. XLVI (1), 2013.

[25] M. Kondo, On the structure of generalized rough sets, Inform. Sci. 176, 589-600, 2006.

[26] M. Kondo and W.A. Dudek, Topological structures of rough sets induced by equivalence relations, J. Advance Comput. Intell. Intell. Inform. 10 (5), 621-624, 2006.

[27] E.F. Lashin, A.M. Kozae, A.A. Abo Khadra, and T. Medhat, Rough set theory for topological spaces, Internat. J. Approx. Reason. 40, 35-43, 2005.

[28] T.J. Li and Y.L. Jing, Rough set model on granular structures and rule induction, Intern. J. Database Theory Appl. 4 (1), 7-18, 2011.

[29] Z. Li, T. Xie, and Q. Li, Topological structure of generalized rough sets, Comput. Math. Appl. 63, 1066-1071, 2012.

[30] T.Y. Lin, Q. Liu, and Y.Y. Yao, Logic systems for approximate reasoning: via rough sets and topology, Methodologies for Intelligent Systems. Berlin, Germany: SpringerVerlag, 1994.

[31] W.J. Liu, Topological space properties of rough sets, in: Proceedings of the Third International Conferences on Machine Learning and Cybernetics, 2353-2355, Shanghai, 2004.

[32] E.G. Manes, Algebraic Theories, Springer, 1976.

[33] B.P. Mathew and S.J. John, On rough topological spaces, Intern. J. Math. Archive 3 (9), 3413-3421, 2012.

[34] T. Medhat, Topological spaces and covering rough sets, Advances Inform. Sci. Service Sci. 3 (8), 283-289, 2011.

[35] K. Monks, A category of topological spaces encoding a cyclic set theoretic dynamics, in: Proceedings of the International Conference on the Collatz Problem and Related Topics, 2000.

[36] S.A. Naimpally and B.D. Warrack, Proximity Spaces, Cambridge Tract in Math. Math. Phys, Cambridge University Press 59, 1970.

[37] P. Pagliani and M. Chakraborty, A Geometry of Approximation, Springer, Dordrecht, 2008.

[38] Z. Pawlak, Information systems-theoretical foundations, Inf. Syst 6 (3), 205-218, 1981.

[39] Z. Pawlak, Rough sets, Int. J. Comput. Inf. Sci. 11 (5), 341-356, 1982.

[40] Z. Pawlak, Rough Sets: Theoretical Aspects of Reasoning About Data, Kluwer Academic Publishers Boston, 1991.

[41] Z. Pawlak and W. Marek, Rough sets and information systems, ICS. PAS. Reports 441, 481-485, 1981.

[42] Z. Pawlak and A. Skowron, Rudiment of rough sets, Inform. Sci. 177, 3-27, 2007. 
[43] J.F. Peters, Near sets. Special theory about nearness of objects, Fund. Inform. 75(3-4), 407-433, 2007.

[44] J.F. Peters, A. Skowron, and J. Stepaniuk, Nearness of objects: Extension of approximation space model, Fund. Inform. 79 (3-4), 497-512, 2007.

[45] J.F. Peters, A. Skowron, and J. Stepaniuk, Nearness of visual objects. Application of rough sets in proximity spaces, Fund. Inform. 128, 159-176, 2013.

[46] L. Polkowski, Rough Sets: Mathematical Foundations, Heidelberg New York, PhysicaVerl., 2002.

[47] L. Polkowski and A. Skowron, Rough sets in Knowledge Discovery 1: Methodology and Applications, Stud. Fuzziness Soft Comput. 18, Physica-Verlag, Heidelberg, Germany, 1998.

[48] L. Polkowski and A. Skowron, Rough Sets in Knowledge Discovery 2: Applications, Case Studies and Software Systems, Stud. Fuzziness Soft Comput. 19, Physica-Verlag, Heidelberg, Germany, 1998.

[49] A.N. Prior, Tense-logic and the continuity of time, Studia Logica 13 (1), 133-148, 1962.

[50] K. Qin and Z. Pei, On the topological properties of fuzzy rough sets, Fuzzy Sets and Systems 151, 601-613, 2005.

[51] K. Qin, J. Yang, and Z. Pei, Generalized rough sets based on reflexive and transitive relations, Inform. Sci. 178, 4138-4141, 2008.

[52] S. Ramanna, A.H. Meghdadi, and J.F. Peters, Nature-inspired framework for measuring visual image resemblance: A near rough set approach, Theoret. Comput. Sci. 412, 5926-5938, 2011.

[53] H. Rasiowa, An Algebraic Approach to Non-Classical Logics, Stud. Logic Found. Math. 78, North-Holland Publishing Company, Amsterdam, London - PWN, Warsaw, 1974.

[54] M. Restrepo, C. Cornelis, and J. Gomez, Characterization of neighborhood operators for covering based rough sets, using duality and adjoining, in: Fourth International Workshop Proceedings Eureka-2013, 98-104, 2013.

[55] A.S. Salama, Some Topological properties of rough sets with tools for data mining, Int. J comp. sci. Issues 8 (2), 588-595, 2011.

[56] A. Skowron, On the topology in information systems, Bull. Pol. Acad. Sci. Math 36, 477-480, 1988.

[57] M.H. Stone, Topological representation of distributive lattices and Brouwerian logics, J. Symb. Log. 3 (2), 90-91, 1938.

[58] A. Tarski, Grundzüge der Syntemenkalküls I, Fund. Math. 25 (1), 503-526, 1935.

[59] M.L. Thivagar, C. Richard, and N.R. Paul, Mathematical innovations of a modern topology in medical events, Int. J. inform. Sci. 2 (4), 33-36, 2012.

[60] N.D. Thuan, Covering rough sets from a topological point of view, Int. J. Comp. Theory Eng. 1 (5), 606-609, 2009.

[61] S. Tiwari, Ultrafilter completeness in ع-approach nearness Spaces, Math. Comput. Sci. 7, 107-111, 2013.

[62] S. Tiwari and P.K. Singh, An approach of proximity in rough set theory, Fund. Inform. 166 (3), 251-271, 2019.

[63] S. Tiwari and P.K. Singh, Čech rough proximity spaces, Mat. Vesnik, accepted, 2019.

[64] M. Vlach, Algebraic and topological aspects of rough set theory, in: IEEE Fourth International Workshop on Computational Intelligence and Application 2008 (1), 23-30, 2008.

[65] S. Wang, P. Zhu, and W. Zhu, Structures of covering-based rough sets, Int. J. Math. Computer Sci. 6 (3), 147-150, 2010.

[66] A. Wasilewska, Topological Rough Algebras, Rough sets and data mining. Springer, Boston MA, 411-425, 1997.

[67] A. Wiweger, On topological rough sets, Bull. Pol. Acad. Sci. Math. 37, 51-62, 1988. 
[68] M. Wolski, Formal concept analysis and rough set theory from the perspective of finite topological approximations, in: Transaction on Rough Sets III, LNCS 3400, 230-243, 2005.

[69] M. Wolski, Rough sets in terms of Discrete Dynamical system, International Conference on rough Sets and Current Trend in Computing 2010, 237-246, 2010.

[70] M. Wolski, Granular computing: Topological and categorical aspects of near and rough set approaches to granulation of knowledge, in: Transaction on Rough Sets XVI, LNCS 7736, 34-52, 2013.

[71] W.Z. Wu, Study on relationship between fuzzy rough approximation operators and fuzzy topological spaces, International Conference on Fuzzy Systems and Knowledge Discovery, LNCS 3613, 167-174, 2005.

[72] W.Z. Wu and J.S. Mi, Some mathematical structures of generalized rough sets in infinite universes of discourse, in: Transactions on Rough Sets XIII, LNCS 6499, 175-206, 2011.

[73] Q.E. Wu, T. Wang, Y.X. Huang, and J.S. Li, Topology theory on rough sets, IEEE Transactions on Systems, man and Cybernetics, Part B (Cybernetics) 38 (1), 68-77, 2008.

[74] T. Yang, Q. Li, and B. Zhou, On the topological structures of granular reducts with covering rough sets, IEEE International Conference on Granular Computing, 601-604, 2012.

[75] Y.Y. Yao, Constructive and algebraic methods of the theory of rough sets, Inform. Sci. 109 (1-4), 21-47, 1998.

[76] Y.Y. Yao, Relational interpretations of neighborhood operator and rough set approximation operators, Inform. Sci. 111 (1-4), 239-259, 1998.

[77] Y.Y. Yao and T.Y. Lin, Generalization of rough sets using modal logic, Intell. Autom. Soft Co. 2 (2), 103-120, 1996.

[78] Y.Y. Yao and B. Yao, Covering based rough sets approximations, Inform. Sci. 200, 91-107, 2012.

[79] Y.Y. Yao and N. Zhong, Potential applications of granular computing in knowledge discovery and data mining, in: Proceedings of World Multiconference on Systemics, Cybernetics and Informatics 5, 573-580, 1999.

[80] W. Zakowski, Approximations in the space (U,П), Demonstr. Math. 16 (3), 761-769, 1983.

[81] Y.L. Zhang, J. Li, and C. Li, Topological structure of relational-based generalized rough sets, Fund. Inform. 147 (4), 477-491, 2016.

[82] N. Zhong, J.Z. Dong, and S. Ohsuga, Using rough sets with heuristics to feature selection, J. Intell. Inf. Syst. 16 (3), 199-214, 2001.

[83] N. Zhong, Y. Yao, and M. Ohshima, Peculiarity oriented multidatabase mining, IEEE T. Knowl. Data Eng. 15 (4), 952-960, 2003.

[84] F. Zhu, On Covering Generalized Rough Sets, Master's thesis, The University of Arizona, Tucson, AZ, USA, May 2002.

[85] W. Zhu, Topological approaches to covering rough sets, Inform. Sci. 177 (6), 1499$1508,2007$.

[86] W. Zhu and F.Y. Wang, Reduction and axiomization of covering generalized rough sets, Inform. Sci. 152, 217-230, 2003.

[87] W. Zhu and F.Y. Wang, Covering based granular computing for analysis of conflict, Lecture Notes in Comput. Sci 3975, 566-571, 2006.

[88] W. Zhu and F.Y. Wang, Topological properties in covering-based rough sets, Fourth International Conference on Fuzzy Systems and Knowledge Discovery 1, 289-293, 2007. 